\title{
A COMPREHENSIVE STUDY ON THE INFLUENCE OF RESOLVING AN INJECTOR ORIFICE AND THE INFLUENCE OF CREATING STRIPPED OFF DROPLETS ON SPRAY FORMATION USING THE VSB2 SPRAY MODEL
}

\author{
Vignesh Pandian Muthuramalingam, Andreas Nygren, $\mathcal{E}$ \\ Anders Karlsson* \\ Department of Mechanics and Maritime Sciences, Chalmers University of \\ Technology, Göteborg, Sweden \\ *Address all correspondence to: Anders Karlsson, Department of Mechanics and \\ Maritime Sciences, Chalmers University of Technology, Göteborg, Sweden, \\ E-mail: anders.karlsson@chalmers.se
}

Original Manuscript Submitted: 3/23/2020; Final Draft Received: 11/13/2020

\begin{abstract}
With respect to simulating fuel sprays applied to direct injection engines, few studies in literature have investigated resolving the injector orifice and how this may influence spray predictions for high pressure and temperature diesel engine-like conditions. In this work, we used the stochastic blob and bubble (VSB2) spray model to conduct simulations in which fuel is injected into a constant volume combustion vessel. The injector orifice is resolved into nine cells. The boundary conditions were the same as the Engine Combustion Network (ECN) noncombusting case for n-dodecane (ECN, 2020). Two simulation meshes were compared with experimental data: (1) injector orifice resolved and (2) injector orifice unresolved (grid cells in the orifice region equal orifice diameter). The resolved orifice mesh showed a liquid penetration length slightly higher and closer to experimental values. Spray predictions for an asymmetrical injection velocity was compared for both meshes. Finer structures near the leading edge of the spray (for mixture fraction and temperature fields) seen in the resolved mesh were missing in the unresolved mesh. Resolving the orifice requires a change in the core of the mesh, which also influences the results. The influence of creating new child blobs (liquid parcels are referred to as blobs in this work) stripped off from a parent blob by secondary breakup was also studied. The simulation results suggested that for high pressure and temperature diesel engine-like conditions, the influence of creating new child blobs is insignificant.
\end{abstract}

KEY WORDS: CFD, sprays, numerical model, diesel engine conditions, spray chamber, resolving injector orifice, secondary breakup, stripped off mass

\section{INTRODUCTION}

To reduce dependence on fossil fuels for powering transport vehicles, increasing efforts are being taken to use alternative renewable fuels. In order to use renewable fuels in existing engines, it is important to understand how the thermophysical properties of the fuel affect spray formation and combustion. Two different methods can be used to study fuel sprays: computer simulations 


\section{NOMENCLATURE}

\begin{tabular}{|c|c|c|c|}
\hline$A$ & area of cross section of injector & $P$ & gas pressure \\
\hline & orifice & $P_{\mathrm{sat}, i}$ & saturation pressure for fuel \\
\hline$C_{D}$ & discharge coefficient of nozzle & & component $i$ \\
\hline$D_{B}$ & diameter of blob & $\dot{S}_{E}$ & enthalpy source term from spray \\
\hline$D_{\text {blob }}$ & diameter of blob before secondary & & model \\
\hline$D_{\text {blob }}^{\prime}$ & $\begin{array}{l}\text { breakup } \\
\text { blob diameter after secondary }\end{array}$ & $\dot{S}_{I}$ & $\begin{array}{l}\text { momentum source term from spray } \\
\text { model }\end{array}$ \\
\hline$D_{\text {bub }}$ & $\begin{array}{l}\text { breakup } \\
\text { diameter of bubble in a given grid }\end{array}$ & $\dot{S}_{M}$ & $\begin{array}{l}\text { evaporation source term from spray } \\
\text { model }\end{array}$ \\
\hline & cell & Sh & Sherwood number \\
\hline$D_{e}$ & $\begin{array}{l}\text { effective diameter of droplets in a } \\
\text { blob }\end{array}$ & $T_{\text {blob }, i j}$ & $\begin{array}{l}\text { liquid temperature in the blob for } \\
\text { mass package } i \text { and fuel component } j\end{array}$ \\
\hline$D_{j}$ & $\begin{array}{l}\text { diffusion coefficient for fuel } \\
\text { component } j\end{array}$ & $T_{\mathrm{eq}}$ & $\begin{array}{l}\text { equilibrium temperature of remaining } \\
\text { blob after evaporation has occurred }\end{array}$ \\
\hline$D_{s}$ & stable blob diameter & $T_{f}$ & film temperature \\
\hline $\begin{array}{l}d t_{s} \\
f\end{array}$ & $\begin{array}{l}\text { subtime-step } \\
\text { correction factor that accounts for }\end{array}$ & $T_{\text {guess }}$ & $\begin{array}{l}\text { guess temperature to begin iteration } \\
\text { for calculation of temperature }\end{array}$ \\
\hline & influence of heat transfer on mass & $\Delta t$ & computational time-step \\
\hline & transfer coefficient & $U$ & velocity of injected liquid blob \\
\hline$h$ & enthalpy of gas & $U_{\text {eq }}$ & equilibrium velocity of blob after \\
\hline$h_{i}\left(T_{\text {blob }}\right)$ & $\begin{array}{l}\text { liquid enthalpy of fuel component } \\
i \text { at blob temperature } \\
\text { number of fuel components in the }\end{array}$ & $V_{R}$ & $\begin{array}{l}\text { evaporation has occurred } \\
\text { relative velocity between blob and } \\
\text { the gas }\end{array}$ \\
\hline & multicomponent fuel mixture & We & Weber number \\
\hline$k$ & turbulent kinetic energy of gas & $\mathrm{We}_{\mathrm{cr}}$ & critical Weber number \\
\hline $\begin{array}{l}l_{\text {diff }} \\
m\end{array}$ & $\begin{array}{l}\text { turbulent length scale } \\
\text { mass of gas in a computational }\end{array}$ & $X_{\mathrm{liq}, i}$ & $\begin{array}{l}\text { mole fraction of fuel component } i \text { in } \\
\text { the liquid droplet }\end{array}$ \\
\hline$\dot{m}$ & $\begin{array}{l}\text { grid cell } \\
\text { input mass flow rate profile }\end{array}$ & $y_{\text {fu, sat }, i}$ & $\begin{array}{l}\text { saturated fuel mass fraction for } \\
\text { fuel component } i\end{array}$ \\
\hline$m_{\mathrm{eq}}$ & $\begin{array}{l}\text { mass that is left behind in a blob } \\
\text { after evaporation }\end{array}$ & $Y_{\max , j}$ & $\begin{array}{l}\text { maximum evaporated mass until } \\
\text { saturation for fuel component } j\end{array}$ \\
\hline$m_{\text {evap }, i j}$ & $\begin{array}{l}\text { evaporated mass for mass } \\
\text { package } i \text { and fuel component } j\end{array}$ & $Y_{\mathrm{sc}, j}$ & $\begin{array}{l}\text { supercritically transferred mass for } \\
\text { fuel component } j\end{array}$ \\
\hline$m_{s}$ & $\begin{array}{l}\text { liquid mass stripped off from } \\
\text { parent blob due to secondary }\end{array}$ & Greek & Symbols \\
\hline & breakup & $\epsilon$ & turbulent dissipation energy of gas \\
\hline$N_{D}$ & $\begin{array}{l}\text { actual number of droplets in a } \\
\text { blob }\end{array}$ & $\lambda$ & $\begin{array}{l}\text { thermal conductivity of the liquid } \\
\text { film }\end{array}$ \\
\hline$N_{f}$ & number of fuel components in the & $\mu_{t}$ & turbulent viscosity of gas \\
\hline & multicomponent fuel mixture & $\tau_{\text {breakup }}$ & secondary breakup time constant \\
\hline$N_{\text {rep }}$ & $\begin{array}{l}\text { representative number ofdroplets } \\
\text { in a blob }\end{array}$ & $\tau_{m, i j}$ & $\begin{array}{l}\text { evaporation time constant for mass } \\
\text { package } i \text { and fuel component } j\end{array}$ \\
\hline $\mathrm{Nu}$ & Nusselt number & $\tau_{T, i j}$ & heat transfer time constant for mass \\
\hline Oh & Ohnesorge number & & package $i$ and fuel component $j$ \\
\hline
\end{tabular}


using fuel spray models and experimental investigations under laboratory conditions. This paper focuses on computational fluid dynamics (CFD) simulations and modeling of direct injection fuels sprays.

The most common approach followed in numerical simulations of sprays is to use the discrete droplet model (DDM) (Ducowicz, 1980) for solving the liquid and gas phases. The DDM uses an Eulerian-Lagrangian approach. The gas phase is treated in an Eulerian framework, where conservation equations are solved for specified grid points. In contrast, the liquid phase is treated in a Lagrangian framework, where liquid parcels are tracked in time and space. Each parcel consists of number of droplets with identical properties (e.g., size, temperature, velocity) interacting with the surrounding gas. Identical droplets are grouped into parcels to reduce computational effort. The spray model solves for the parcel position, mass, momentum, and energy. Source terms are obtained by solving the conservation equations for the liquid phase. The source terms are then coupled to the gas phase equations to complete the interaction between the two phases. An alternative approach to using computational parcels to group identical droplets divides the droplets in each computational parcel into a number of bins based on their size distribution. This approach is used when it is desired to use parcels that have a size distribution for the droplets based on the local conditions surrounding it. In the present work, the latter approach is used, and the parcels comprising a size distribution of droplets are referred to as blobs.

Blended fuels are gaining importance in the automotive industry owing to increasingly stringent legislations. Blended fuels are multicomponent mixtures containing conventional diesel or gasoline blended with renewable fuels. In multicomponent fuels, differential evaporation plays an important role as each component evaporates at its own rate. The evaporation of each component changes the gas temperature, which in turn affects the evaporation of other components. These conditions pose challenges when accounting for multicomponent fuels in spray models. Multicomponent fuel spray models can be divided into two categories: discrete [e.g., Ra and Reitz (2009)] and continuous [e.g., Lippert and Reitz (1997)]. In discrete models, each fuel component is tracked separately. Discrete models are computationally expensive but facilitate coupling to reaction mechanisms. On the other hand, in continuous multicomponent models, the mole fraction of each species is represented by a distribution function (with regard to molecular weight), and only equations for the mean and variance of the distribution function are solved. Although continuous models are computationally efficient, the modeling effort is larger than for a discrete model. The spray model used in the present work was a discrete multicomponent model called the stochastic blob and bubble (VSB2) model. Further details of the model can be found in Section 2. The main difference of VSB2 from the abovementioned models is that it uses thermodynamic equilibrium to calculate mass and heat transfer, ensuring there are no overshoots or undershoots in mass transfer or temperature.

To resolve velocity and mixing gradients in spray simulations accurately, it is important to employ sufficiently fine grid cells near the injector orifice. The aforementioned criteria regarding grid cells is sometimes not strictly followed, and underresolved grid cells are used in the orifice region and droplet momentum is coupled with the surrounding gas based on gas jet theory calculations [e.g., see Abani et al. (2008) and Perini and Reitz (2016)]. The idea behind this approach is that underresolved grid cells can still be used if accurate coupling is attained between the liquid and gas phases. In gas jet simulations, the restriction on minimum grid size is removed, as there is no liquid phase. However, two-phase simulations for fine grid cells in the orifice region are still possible if the predictions of spray submodels are as good for fine grid cells as they are for coarser grid cells. The following paragraph discusses the importance, challenges, and introduction to the present work on resolving the injector orifice.

Volume 31, Issue 4, 2021 


\subsection{Resolving the Injector Orifice}

The need for adequate resolution near the injector orifice region has been much discussed in literature. Grid sensitivity analysis was carried out by Kösters and Karlsson (2016). Grid sizes were analyzed from coarse to fine, relative to the orifice diameter. It was shown that grid cells with the size of the order of the orifice diameter are required to achieve a good prediction of spray when compared with experimental data. In another work, a comprehensive grid sensitivity study was performed by Senecal et al. (2012), who concluded that beyond a certain grid size, the accuracy of predicting sprays converges (in this case with regard to liquid penetration length) and resolving the grid cells further does not produce any further significant difference in the liquid penetration length. Senecal et al. (2012) used adaptive mesh refinement (AMR) to refine cells in the spray region. AMR is useful when it is not known beforehand where finer grid cells are required.

Apart from considering grid resolution in the near orifice region, it has been shown that it is important to resolve the injector orifice itself to improve predictions of gas jets. Abraham (1997) performed simulations with resolved injector orifice and suggested that at least two cells are needed across the injector orifice for good prediction of gas jets. The accuracy of jet prediction was gauged by mixing rate and gas velocity fields. Spray simulations were not conducted by Abraham owing to computational limitations, but it was suggested that the implications could also be extended to sprays. To date, few studies have examined the influence of resolving injector orifice for sprays under high pressure and temperature diesel engine-like conditions. Therefore, in the present work, the authors conducted spray simulations for an injector orifice that was divided into nine cells (three cells across the injector diameter) for high pressure and temperature diesel engine precombustion conditions. This is a first study by the authors on resolving injector orifice and was started by choosing nine cells to resolve the orifice. Nine cells were chosen with the idea of having at least one core cell consisting high liquid volume fraction, which is then surrounded by cells interacting with the surrounding gas. The main objectives in resolving the injector orifice were as follows:

- Determine whether it is possible to perform Eulerian (gas phase) Lagrangian (liquid phase) coupling for fine grid cells used in the resolved orifice case, where the liquid fraction in the grid cell is high.

- Assess the influence of resolving the injector orifice on spray formation (mixing of fuel with air, gas phase velocity, liquid and vapor penetration).

- Assess the capability of predicting sprays with an asymmetrical injection velocity.

- Ease the adoption of large eddy simulation for sprays.

\subsection{Creating New Child Blobs from Stripped Off Mass}

The second part of the study concerned stripped off mass. Stripped off mass is the liquid mass that is removed from the parent blob by secondary breakup (discussed further in Section 2.3). If the stripped off droplets are retained in the parent blob, the smaller stripped off droplets in the liquid blob transfer the same momentum as the bigger droplets. Creating new blobs from stripped off droplets is more accurate in terms of momentum transfer. A few studies have examined the influence of stripped off mass [e.g., Karlsson (1995)]. The influence of stripped off droplets was discussed briefly in Kösters and Karlsson (2016), where it was suggested that not accounting 
for stripped off droplets could create an issue in the momentum coupling (drag) near the nozzle where the influence of stripping off is the strongest. Thus the present study investigated whether or not creating new computational blobs from stripped off droplets will make a difference under high pressure and temperature conditions of diesel sprays by comparing simulation results with experimental data.

An introduction to the spray model used in this work is given in the next section. Following that computational setup, solution verification and results are discussed. The results are divided into three parts: influence of resolving the injector orifice, influence of creating stripped off droplets, and a discussion of the influence of using bubble (the concept of bubble is explained in Section 2.9). A short discussion on numerical accuracy is also presented toward the end.

\section{MODEL DESCRIPTION}

The spray model used for simulations was VSB2. The model was developed by Karlsson, and its first implementation and validation with experimental data are described in Karlsson et al. (2008). It is a Lagrangian model solving for the liquid phase. In contrast with the traditional Eulerian-Lagrangian approach, where the liquid fuel is represented by computational parcel containing identically sized droplets, the liquid fuel in the VSB2 model is represented by computational blobs. In each blob, the droplets are divided into a number of bins based on their size distribution governed by a distribution function. The parameters of the distribution function are determined by the local conditions around each specific liquid blob, at a given time instant. The VSB2 model solves primarily for mass, momentum, and energy transfer between the liquid phase and the surrounding gas. Relaxation equations are used to solve mass and heat transfer. The following sections describe some of the important physical processes modeled in VSB2. For further details, refer to Kösters and Karlsson (2016).

\subsection{Blob Creation}

Liquid blobs are injected with a given blob diameter $D_{\text {blob }}$ and a blob mass $m_{\text {blob }}$ given by Eq. (1),

$$
m_{\text {blob }}=N_{\text {rep }} \rho_{\text {liq }} \frac{\pi}{6} D_{\text {blob }}^{3}
$$

where $N_{\text {rep }}$ is a representative number used to maintain mass balance for a given blob with diameter $D_{\text {blob. }} N_{\text {rep }}$ is constant throughout the lifetime of a blob.

\subsection{Secondary Breakup}

The size of a blob as a result of secondary breakup is given by Eq. (2),

$$
D_{\text {blob }}^{\prime}=D_{s}+\left(D_{\text {blob }}-D_{s}\right) e^{-\Delta t / \tau_{\text {breakup }}}
$$

where $D_{\text {blob }}^{\prime}$ is the new blob diameter after breakup, $D_{\text {blob }}$ is the initial blob diameter, $D_{s}$ is the stable droplet diameter, and $\Delta t$ is the computational time-step. $\tau_{\text {breakup }}$ is breakup time, obtained from the correlations of Pilch and Erdman (1987). $D_{s}$ is given by Eq. (3):

$$
D_{s}=\mathrm{We}_{\mathrm{cr}} \sigma /\left(\rho_{\mathrm{gas}} U_{\mathrm{rel}}^{2}\right)
$$

The critical Weber number $\mathrm{We}_{\mathrm{cr}}$ is given by Eq. (4),

$$
\mathrm{We}_{\mathrm{cr}}=12\left(1+1.077 \mathrm{Oh}^{1.6}\right)
$$

Volume 31, Issue 4, 2021 
where Oh represents the Ohnesorge number, which is a function of the Reynolds and Weber numbers.

\subsection{Stripped Off Mass}

The stripped off mass is the liquid mass removed from the parent blob by secondary breakup. It is given by Eq. (5),

$$
m_{s}=\rho_{\text {liq }} \frac{\pi}{6}\left(D_{\text {blob }}^{3}-D_{\text {blob }}^{\prime 3}\right)
$$

where $D_{\text {blob }}$ and $D_{\text {blob }}^{\prime}$ are the size of blob before and after breakup, respectively.

\subsection{Droplet Size Distribution}

The size distribution of droplets in a blob is represented by a one-parameter cumulative distribution function (CDF), where the parameter $f$ is called power coefficient $f$. The CDF takes the form given by Eq. (6),

$$
D=M^{f}
$$

where $D$ and $M$ correspond to the normalized droplet size and normalized droplet mass, respectively. The droplet size is normalized by $D_{\text {blob }}$ (parent blob diameter), and droplet mass is normalized by the parent blob mass (mass corresponding to a droplet of size $D_{\text {blob }}$ ). To calculate the power coefficient $f$, we take a calculated value for $D$ and $M$, namely the stable droplet diameter and the stripped off mass. The normalized stable droplet diameter is given by Eq. (7):

$$
D=\frac{D_{s}}{D_{\text {blob }}}
$$

The normalized stripped off mass is given by Eq. (8):

$$
M=\frac{D_{\text {blob }}^{3}-D_{\text {blob }}^{\prime 3}}{D_{\text {blob }}^{3}}=1-\frac{D_{\text {blob }}^{\prime 3}}{D_{\text {blob }}}
$$

The power coefficient is then given by Eq. (9):

$$
f=\frac{\log \left(D_{s} / D_{\text {blob }}\right)}{\log \left[1-\left(D_{\text {blob }}^{\prime} / D_{\text {blob }}\right)^{3}\right]}
$$

The normalized droplet mass [ $M$ in Eq. (6)] is divided into 10 mass packages. The first mass package (or first size interval) is given by the stripped off mass. The remaining mass is divided into nine equal mass packages (intervals). The size of each interval is calculated from the corresponding mass using Eq. (6). The droplet size distribution for three values of $f$ is shown in Fig. 1.

\subsection{Mass Transfer}

Mass transfer from the liquid to the gas phase is given by Eq. (10),

$$
\frac{d m_{\mathrm{evap}, i, j}}{d t}=\frac{m_{\mathrm{eq}, j}-m_{\mathrm{blob}, i, j}}{\tau_{m, i, j}}
$$




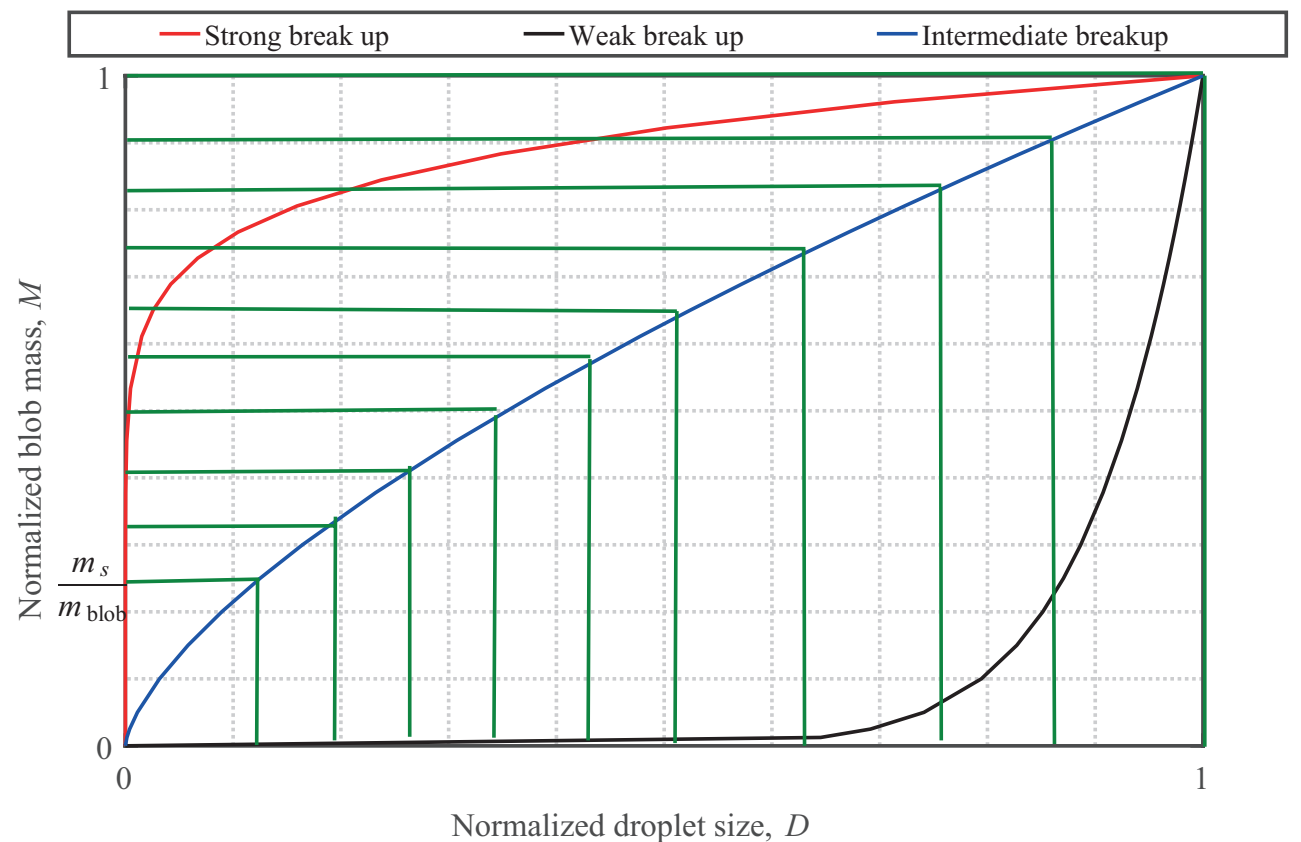

FIG. 1: Cumulative droplet size distribution function

where $i$ denotes a specific mass package and $j$ denotes a specific fuel component (in a multicomponent fuel mixture) for a given blob, $m_{\mathrm{eq}, j}$ is the equilibrium mass for fuel component $j$ (the mass evaporated under the given conditions until the gas is saturated, discussed further in Section 2.8), and $\tau_{m, i, j}$ is the evaporation time scale. $\tau_{m, i, j}$ is given by Eq. (11),

$$
\tau_{m, i, j}=\frac{\rho_{l} D_{\mathrm{blob}, i}^{2} R T_{f}\left(Y_{\mathrm{eq}, j}-Y_{\mathrm{sc}, j}\right)}{6 P D_{j} S h}
$$

where $\rho_{l}$ is liquid density, $D_{\mathrm{blob}, i}$ is the diameter defined for size interval (mass package) $i, T_{f}$ is film temperature, $Y_{\mathrm{eq}, j}$ is the maximum evaporated liquid mass fraction (fraction of blob mass) until saturation for fuel component $j, Y_{\mathrm{sc}, j}$ is supercritically transferred mass fraction for fuel component $j, D_{j}$ is diffusion coefficient for fuel component $j$, and Sh is Sherwood number.

The mass transferred when the gas temperature and pressure are above the critical values is independent of time as it is assumed to be an instantaneous process.

\subsection{Heat Transfer}

The heat transfer to the blob is given by Eq. (12),

$$
\frac{d T_{\text {blob }}}{d t}=\sum_{i=1}^{n_{\text {blob }}} \frac{T_{\text {eq }}-T_{\text {blob }}}{\tau_{T, i}}
$$

where $n_{\text {blob }}$ is the number of size intervals (mass packages) and $T_{\text {eq }}$ is the equilibrium temperature of the blob-bubble system. It is calculated from the energy balance after mass transfer has

Volume 31, Issue 4, 2021 
occurred. $T_{\text {blob }}$ is the temperature of mass package $i$ and $\tau_{T, i}$ is heat transfer time scale. It is given by Eq. (13):

$$
\tau_{T, i}=\frac{\rho_{l} D_{\text {blob }, i}^{2} C_{p, \text { blob }}}{6 \lambda \mathrm{Nu} f}
$$

$\lambda$ is the thermal conductivity of the liquid film, $f$ is the correction factor that accounts for influence of heat transfer on mass transfer coefficient as given by El Wakil et al. (1954), and $\mathrm{Nu}$ is the Nusselt number.

\subsection{Momentum Transfer}

The momentum equation for the blob is given by Eq. (14),

$$
m_{\text {blob }} \frac{d U_{\text {blob }}}{d t}=\sum_{i=1}^{n_{\text {blob }}} \frac{\pi D_{i}^{2}}{8} \rho_{g} C_{d} V_{R}\left(U_{g}^{\prime}-U_{\text {blob }}^{\prime}\right)
$$

where subscript $i$ refers to a specific size interval, $D_{i}$ is the diameter of droplet belonging to size interval $i, V_{R}$ is the relative velocity at the old time-step

$$
V_{R}=\left|U_{g}^{o}-U_{\text {blob }}^{o}\right|
$$

and $U_{g}^{\prime}$ and $U_{\text {blob }}^{\prime}$ are the gas and blob velocities at the new time-step. $C_{d}$ in Eq. (14) is the drag coefficient; $C_{d}$ at Reynolds numbers between 5 and 2000 is given by Eq. (16) as suggested by Schmehl et al. (2000).

$$
\begin{aligned}
C_{d} & =0.28+\frac{21}{\operatorname{Re}_{i}}+\frac{6}{\sqrt{\operatorname{Re}_{i}}}+\mathrm{We}\left[0.2319-0.1579 \log \left(\operatorname{Re}_{i}\right)\right. \\
& \left.+0.0471 \log ^{2}\left(\operatorname{Re}_{i}\right)-0.0042 \log ^{3}\left(\operatorname{Re}_{i}\right)\right]
\end{aligned}
$$

The equation for $C_{d}$ accounts for droplet deformation in the subcritical Weber number range. At higher Reynolds numbers, $C_{d}$ is equal to 0.424 . The momentum transferred from the liquid blob to the gas is given by Eq. (17):

$$
m_{g} \frac{d U_{g}}{d t}=-m_{\text {blob }} \frac{d U_{\text {blob }}}{d t}
$$

Substituting Eq. (17) in Eq. (14) gives an expression for the blob velocity at the new time-step.

\subsection{Calculation of Equilibrium Mass}

The equilibrium mass is the mass of liquid that can evaporate under given pressure and temperature conditions until the surrounding gas is saturated. A schematic of the evaporation of a blob is given in Fig. 2.

Evaporation of each fuel component takes place until its saturated mass fraction is reached. The saturated fuel mass fraction is calculated from Eq. (18),

$$
Y_{\mathrm{fu}, \mathrm{sat}, i}=\frac{M_{\mathrm{fu}, i}}{M_{\mathrm{mix}}} \frac{P_{\mathrm{sat}, i}\left(T_{\mathrm{bub}}\right)}{P} X_{\mathrm{liq}, i}
$$




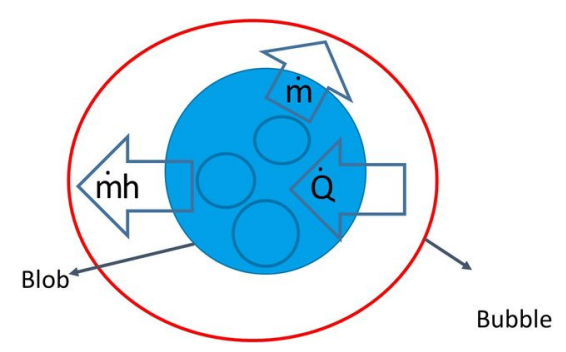

FIG. 2: Evaporation of blob in a bubble

where $Y_{\mathrm{fu}, \mathrm{sat}, i}$ is the saturated mass fraction for fuel component $i, M_{\mathrm{fu}, i}$ is the molecular weight of fuel component $i, M_{\mathrm{mix}}$ is the molecular weight of the mixture in the gas phase, $P_{\mathrm{sat}, i}$ is the saturated vapor pressure of fuel component $i, P$ is the gas pressure, $T_{\text {bub }}$ is the bubble temperature, and $X_{\mathrm{liq}, i}$ is the mole fraction of fuel component $i$ in the liquid droplet.

Due to evaporation, mass and enthalpy are transferred to the bubble. The change in the mass fraction and enthalpy of the bubble in turn results in a change in the bubble temperature. Thus evaporation alters the mass fraction of all species, bubble enthalpy, and temperature. These processes are represented by Eqs. (19)-(21),

$$
\begin{gathered}
Y_{i}^{\prime}=\frac{y_{i} m+\Delta m_{i}}{m+\sum_{1}^{N_{f}} \Delta m_{i}} \\
h_{g}^{\prime}=\frac{m h_{g}+\sum_{1}^{N_{f}} \Delta m_{i}\left[h_{\text {fuel, }, \mathrm{vap}, i}\left(T_{d r}\right)-\Delta h_{\mathrm{vap}, i}\left(T_{d r}\right)\right]}{m+\sum_{1}^{N_{f}} \Delta m_{i}} \\
h_{g}^{\prime}(T)=\sum Y_{i}^{\prime} h_{i}(T)
\end{gathered}
$$

where $\Delta m_{i}$ is the mass of fuel component $i$ that has evaporated until saturation; $Y_{i}^{\prime}, h_{g}^{\prime}$, and $T^{\prime}$ are the updated mass fraction, enthalpy, and temperature, respectively, for the gas phase. Equation (21) is a function calculating the gas temperature from the gas enthalpy and mass fraction of species in the gas. The set of nonlinear equations shown above, are solved simultaneously using the SUNDIALS' KINSOL solver (Computing at Lawrence Livermore National Laboratory, 2020). SUNDIALS is a software package that provides time integrators and nonlinear solvers that can be coupled with CFD codes. KINSOL is the solver package in SUNDIALS used to solve nonlinear algebraic equation systems. The variables involved in the system of nonlinear equations representing evaporation are shown in Fig. 3.

The solution to the above-mentioned equations yield the equilibrium mass, which is then used in Eq. (10).

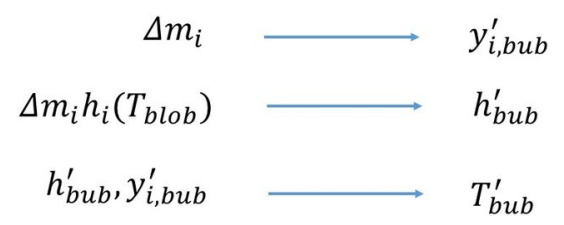

FIG. 3: Variables involved in the nonlinear equations representing evaporation

Volume 31, Issue 4, 2021 


\subsection{The Bubble Approach}

To minimize grid dependency, the blob was assumed to interact with a surrounding volume enclosed by a bubble. The volume of the bubble is less than or equal to the grid cell. For larger grid cells, the volume of the bubble is much smaller than the grid cell. A schematic of the blob and bubble concept is shown in Fig. 4.

The bubble size controls the rate of evaporation in large grid cells. The volume of the bubble is given by Eq. (22),

$$
V_{\text {bub }}=\rho_{g} N_{D} \frac{\pi}{4} D_{e}^{2} l_{\text {diff }}
$$

where $N_{D}$ is the actual number of droplets in the blob, and $D_{e}$ is the effective diameter of the droplets in the blob. It is given by Eq. (23),

$$
D_{e}=\sum_{i=1}^{n_{\text {blob }}} \frac{m_{i}}{m_{\text {blob }}} D_{i}
$$

where $n_{\text {blob }}$ is the number of size intervals and $D_{i}$ is the diameter defined for size interval $i . N_{D}$ is calculated from $D_{e}$, similar to Eq. (1).

$l_{\text {diff }}$ in Eq. $(22)$ is the turbulent length scale. $l_{\text {diff }}$ is based on turbulent diffusion and is given by $\mathrm{Eq}(24)$,

$$
l_{\text {diff }}=\sqrt{\frac{\mu_{t}}{\rho_{g}} d t_{s}}
$$

where $\mu_{t}$ is the turbulent viscosity and $d t_{s}$ is the subtime-step.

\subsection{Turbulence Modeling}

The turbulence model used for the gas phase in this work is the standard two-equation $k-\epsilon$ model. It is used to model the unclosed terms arising from the Reynolds averaged Navier-Stokes equation. This model was originally developed for incompressible flows (Jones and Launder, 1972) and then later modified to describe compressible flows (Goasman and Watkins, 1977). The transport equation for the turbulent kinetic energy $k$, is given by Eq. (25):

$$
\frac{\partial \rho k}{\partial t}+\nabla(\rho k u)=\nabla\left[\left(\frac{\mu_{t}}{\sigma_{k}}+\mu\right) \nabla k\right]+\mu_{t}\left[S-\frac{2}{3}(\nabla u)^{2}\right]-\frac{2}{3} \rho k \nabla u-\rho \epsilon
$$

The transport equation for dissipation of the turbulent energy $€$ is given by Eq. (26),

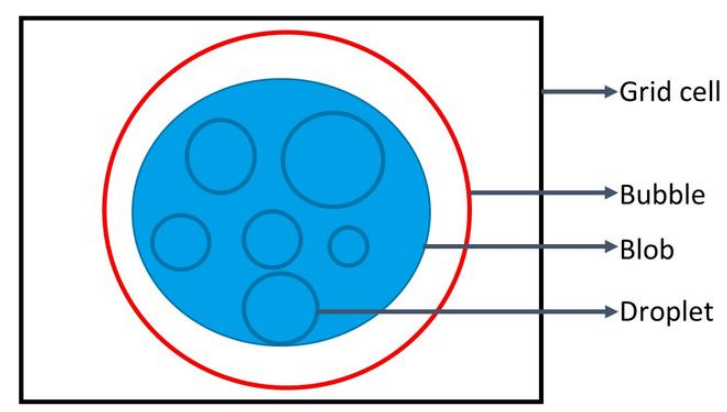

FIG. 4: Blob and bubble concept 


$$
\begin{aligned}
\frac{\partial \rho \epsilon}{\partial t}+\nabla(\rho \epsilon u) & =\nabla\left[\left(\frac{\mu_{t}}{\sigma_{\epsilon}}+\mu\right) \nabla \epsilon\right]+\mu_{t} C_{1} \frac{\epsilon}{k}\left[S-\frac{2}{3}(\nabla u)^{2}\right] \\
& -\frac{2}{3} C_{1} \rho \epsilon \nabla u-C_{2} \rho \frac{\epsilon^{2}}{k}+C_{3} \rho \epsilon \nabla u
\end{aligned}
$$

where $S$ is given by Eq. (27):

$$
S=2 S_{i j} S_{i j}=\frac{1}{2}\left(\frac{\partial u_{j}}{\partial x_{i}}+\frac{\partial u_{i}}{\partial x_{j}}\right)^{2}
$$

The coefficients $C_{1}-C_{3}$ of the $k-\epsilon$ model are optimized for the case under study in accordance with previously established simulations by different simulation groups and presented at an ECN2 workshop (ECN2, 2012). The constants are summarized in Table 1.

\section{COMPUTATIONAL METHODS}

All simulations were performed using OpenFOAM-2.2.x (Weller et al., 1998; OpenFOAM, 2020). The VSB2 spray model was implemented in OpenFOAM. Nonlinear equations for mass transfer were solved using the SUNDIALS KINSOL solver (Computing at Lawrence Livermore National Laboratory, 2020). The solver was coupled to OpenFOAM. The submodels used in the simulations are summarized in Table 2.

It was not in the scope of this work to investigate droplet collisions. Primary breakup of droplets was not taken into account because the blob injection method is followed. A unit injector was used. The main difference between the different injectors in OpenFOAM is the way in which the velocity is calculated. For a unit injector, the velocity is calculated from the input mass flow rate profile as shown in Eq. (28):

$$
U=\frac{\dot{m}}{\rho C_{D} A}
$$

Droplets (contained in blobs) are injected in a disc centered around the injector position with the same diameter as the nozzle orifice. The dispersion of spray droplets by turbulent gas motion is

TABLE 1: Coefficients used for the $k-\epsilon$ model

\begin{tabular}{|l|c|c|c|c|c|}
\hline $\mathbf{C 1}$ & $\mathbf{C 2}$ & $\mathbf{C 3}$ & $\boldsymbol{C}_{\boldsymbol{\mu}}$ & $\boldsymbol{\sigma}_{\boldsymbol{\epsilon}}$ & $\boldsymbol{\sigma}_{\boldsymbol{k}}$ \\
\hline 1.55 & 1.92 & -0.33 & 0.09 & 1.65 & 1 \\
\hline
\end{tabular}

TABLE 2: Submodels used

\begin{tabular}{|l|c|}
\hline Submodel & Name \\
\hline Breakup & Pilch Erdman/relaxation equation \\
\hline Mass transfer & Relaxation equation \\
\hline Heat transfer & Relaxation equation \\
\hline Drag & Drag equation \\
\hline Collision & None \\
\hline Atomization & None \\
\hline Injector model (type of spray) & Solid cone \\
\hline Injector (injector setup) & Stochastic dispersion \\
\hline Dispersion model & $1 \mu \mathrm{s}$ \\
\hline Computational time-step & 0.5 \\
\hline Max Courant number & \\
\hline
\end{tabular}

Volume 31, Issue 4, 2021 
calculated using a stochastic dispersion model (Gosman and Ioannides, 1983). In this model, the turbulent velocity is sampled from a Gaussian distribution with a variance of $\sqrt{2 k / 3}$, where $k$ is the turbulent kinetic energy of the gas.

\subsection{Computational Mesh}

A constant volume cylindrical combustion chamber was meshed using blockMesh, an OpenFOAM tool that decomposes the geometry into hexahedral blocks. The 3D mesh is shown in Fig. 5.

Two mesh setups were studied: one where the injection orifice was not resolved (cell size in the $x$ and $y$ direction of the order of the injector orifice) and another where the orifice was resolved into nine cells. A picture of the resolved injector orifice is shown in Fig. 6 (the orifice is the innermost concentric circle). Top views of both meshes are shown in Fig. 7 (note that the resolved orifice mesh is zoomed in to show the injector orifice).

Both meshes were fine in the central region around the spray and became increasingly coarse toward the cylinder's periphery (the mesh in the central region was much finer for the resolved

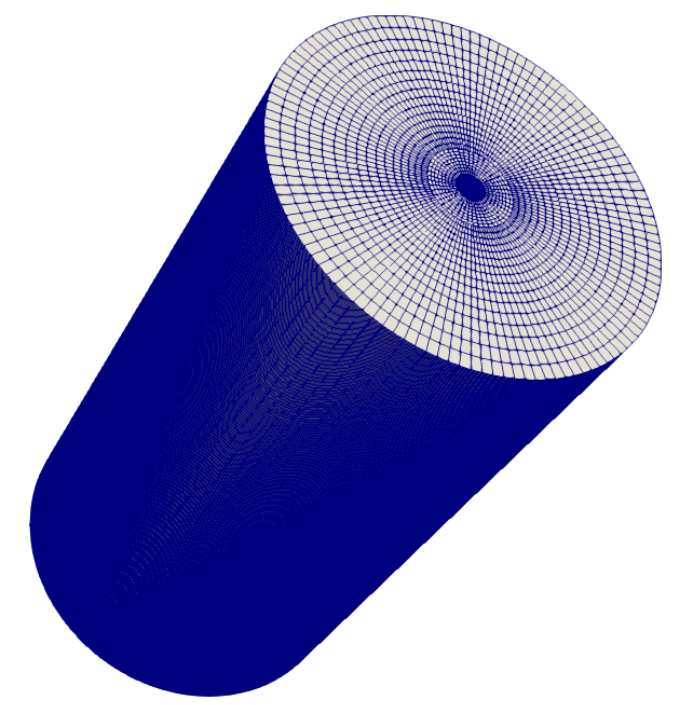

FIG. 5: Combustion chamber mesh

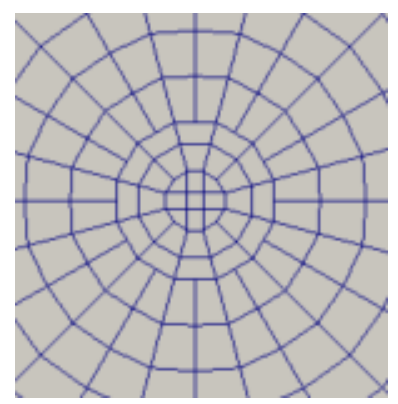

FIG. 6: Conceptual picture of injector orifice 


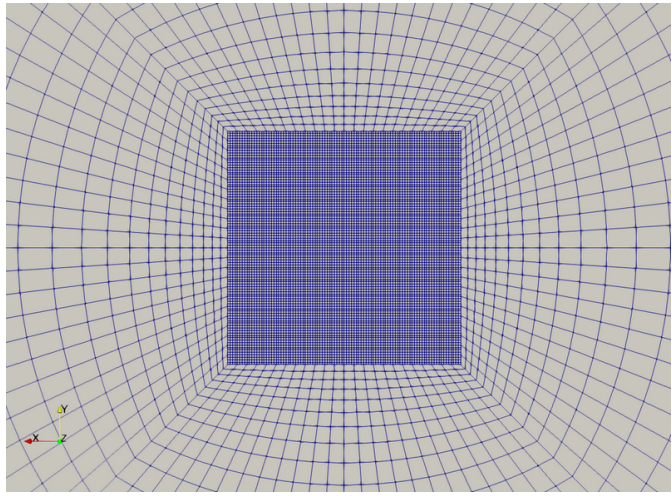

(a)

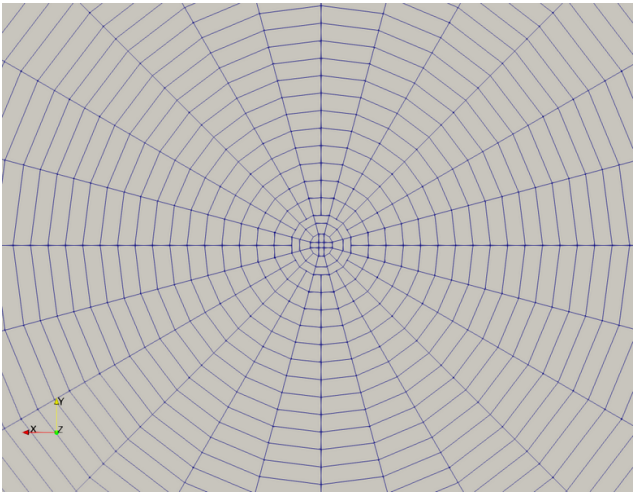

(b)

FIG. 7: Top view of the unresolved and resolved meshes. (a) Unresolved orifice and (b) resolved orifice, zoomed.

orifice). The mesh was refined in the spray region where there are a lot of spray-gas interactions, but not in the periphery as the gas there is more or less at ambient conditions. The nozzle orifice diameter was $84 \mu \mathrm{m}$. In the unresolved mesh, each cell in the refined region had a dimension of $125 \times 125 \times 250 \mu \mathrm{m}$ (a prior grid sensitivity study done by Kösters and Karlsson (2016) identified this grid size to be sufficient for the conditions under investigation). Each cell in the $x-y$ plane was thus of the order of the nozzle orifice diameter, although slightly coarser. In the resolved mesh, the injector orifice was resolved into nine cells (three cells across the diameter). The innermost cell in the core spray region (within the orifice) had a dimension of $28 \times 28 \times$ $62.5 \mu \mathrm{m}$. A cut plane view along the $z$-axis for the resolved mesh is shown in Fig. 8 .

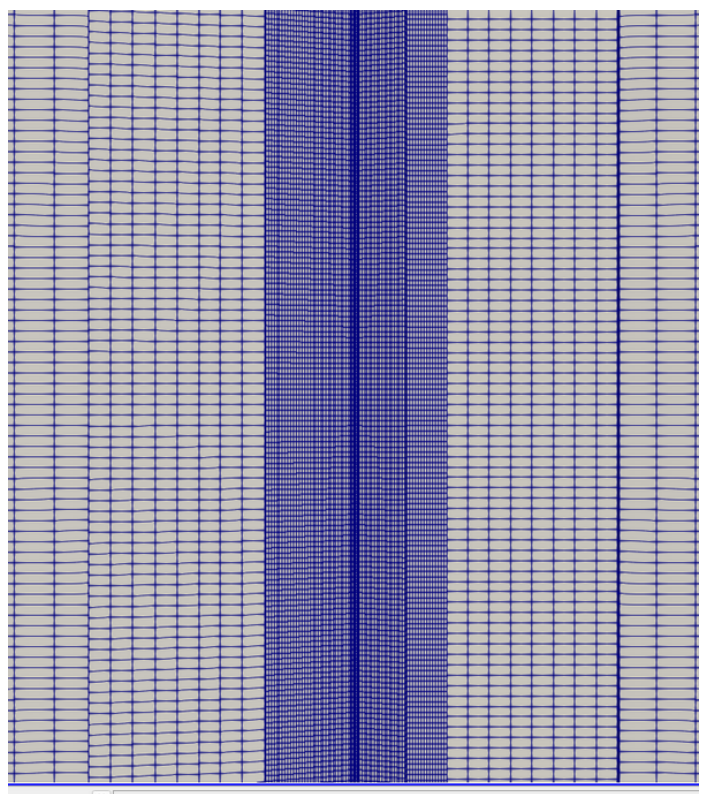

FIG. 8: Cut plane view of mesh along injector $(z)$ axis for the resolved orifice

Volume 31, Issue 4, 2021 
While resolving the injector orifice and injecting blobs with orifice diameter, it is ensured that the blob volume is smaller than that of the grid cell by considering a representative number of blobs as shown in Eq. (29),

$$
\dot{m}_{\text {inj }} \Delta t=\frac{N_{\text {blob }}}{t_{\text {inj }}} \Delta t N_{\text {rep }} \rho_{\text {liq }} \frac{\pi}{6} D_{\text {nozzle }}^{3}
$$

where $\dot{m}_{\text {inj }}$ is the injector mass flow rate, $\Delta t$ is the computational time-step, $N_{\text {blob }}$ is the total number of blobs (set as an input), $t_{\text {inj }}$ is injection duration, $\rho_{l}$ is liquid density, and $N_{\text {rep }}$ is the representative number of blobs [according to Eq. (1)]. Since the mass flow rate is given as input, by adjusting the value of $N_{\text {blob }}, N_{\text {rep }}$ will in turn be adjusted so as to ensure that the blob volume is less than that of the grid cell.

\section{EXPERIMENTAL VALIDATION DATA}

All simulations are compared against the ECN spray A noncombusting (no oxygen) experimental case (ECN, 2020). The institution that provided the experimental data is Sandia. The boundary conditions are summarized in Table 3.

\section{SOLUTION VERIFICATION}

Before discussing validation with experiments, the verification of numerical solution is presented. According to ASME V\&V 20-2009 standard (ASME, 2009): "Solution verification estimates the numerical accuracy of a particular calculation. Solution verification involves error estimation since the exact solution to the specific problem is unknown." It involves performing grid convergence studies on chosen grids obtained by systematic refinement. The study will then yield the uncertainty of the key variables under consideration. Once the solution is verified, the model is then validated against experimental data to determine the degree to which the model is an accurate representation of real world. It should be noted that the solution verification that is done here is intended to provide a general idea of the numerical uncertainty at a specific point in time for a few key variables. Detailed uncertainty analysis is not in the scope of this work.

In order to minimize the verification error, geometrically similar grids are recommended. As Fig. 7 shows, the unresolved mesh used for validation with experiment was intended to have a cartesian core and the resolved mesh had a cylindrical core. However, in order to have geometrically similar grids for the purpose of verification alone, two additional meshes are created for the unresolved orifice, and they have a cylindrical core, similar to the resolved orifice mesh. The two unresolved meshes have dimensions of the innermost cell (surrounding the orifice) as $250 \mu \mathrm{m}$ and $125 \mu \mathrm{m}$, respectively. In total, three meshes are used for verification (shown in Fig. 9, the

TABLE 3: Boundary conditions

\begin{tabular}{|l|c|}
\hline Ambient temperature & $900 \mathrm{~K}$ \\
\hline Ambient pressure & $60.5 \mathrm{bar}$ \\
\hline Injection pressure & $1500 \mathrm{bar}$ \\
\hline Injection duration & $1.5 \mathrm{~ms}$ \\
\hline Total injected mass & $3.46 \mathrm{mg}$ \\
\hline Nozzle diameter & $84 \mu \mathrm{m}$ \\
\hline
\end{tabular}




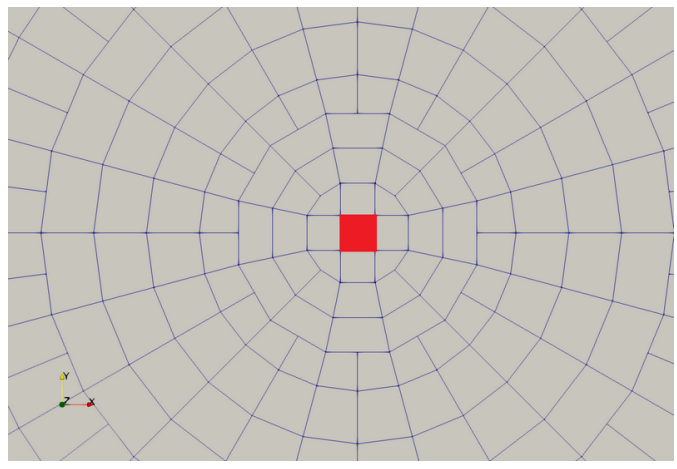

(a)

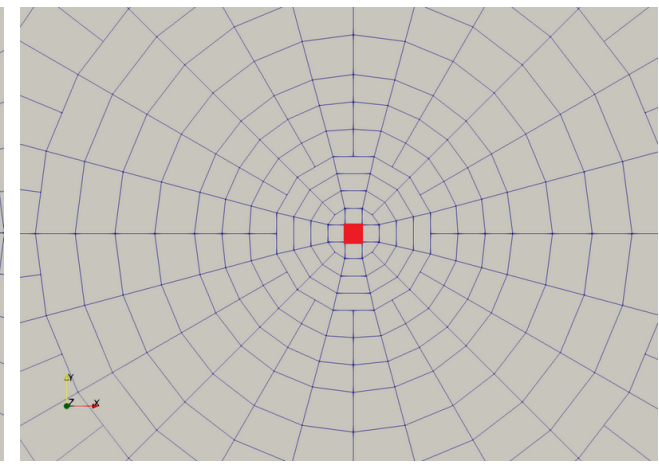

(b)

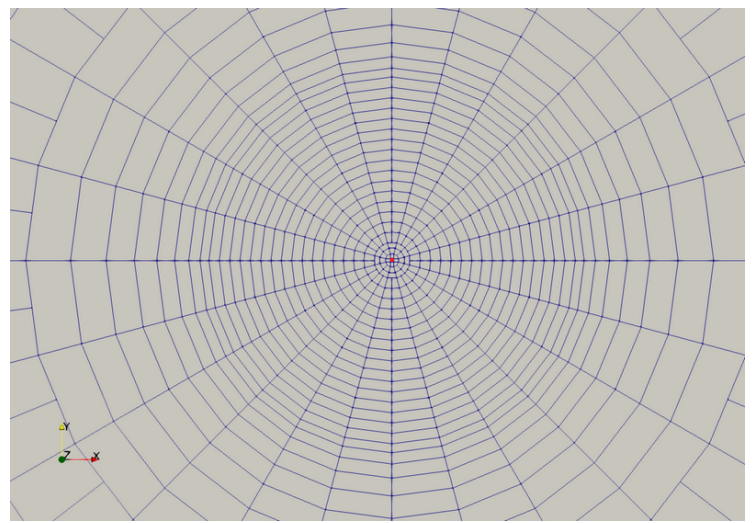

(c)

FIG. 9: Top view of the unresolved and resolved meshes with cylindrical core. Unresolved orifice, (a) $\min$ grid size $=250 \mu \mathrm{m}$; (b) min grid size $=125 \mu \mathrm{m}$; (c) resolved orifice, min grid size $=28 \mu \mathrm{m}$.

squares indicate the grid cell surrounding the orifice, which are also the grid cells with minimum length in the $x$ and $y$ directions).

The solution verification is done following a five-step procedure as suggested by ASME V\&V 20-2009 standard (ASME, 2009). The procedure uses Richardson extrapolation (RE) (Richardson, 1910) to calculate the error of a particular variable under study. Uncertainty estimates are then calculated by Roache's (Roache, 1998) grid convergence index (GCI). The GCI has an estimated $95 \%$ uncertainty (95\% confidence interval) obtained by multiplying the RE error with a factor of safety $F_{s}$. The five-step procedure is summarized below.

Step 1: A representative grid size $h$ is chosen for each mesh. In this case, $h$ is the size of the grid cell surrounding the orifice.

Step 2: Three sets of meshes are chosen as shown earlier (Fig. 9). The key variables for study are vapor penetration, maximum value of axial velocity (from the radial distribution at $25 \mathrm{~mm}$ from the injector), and maximum value of mixture fraction (from the radial distribution at $25 \mathrm{~mm}$ from the injector $)$. The grid refinement factor $r=\left(h_{\text {coarse }} / h_{\text {fine }}\right)$ is calculated.

Volume 31, Issue 4, 2021 
Step 3: The representative grid sizes are chosen such that $h_{1}<h_{2}<h_{3}$ and $r_{21}=h_{2} / h_{1}$, $r_{32}=h_{3} / h_{2}$, and the apparent order of the method, $p$ is calculated by solving Eq. (30)(32) (Roache, 1998).

$$
\begin{gathered}
p=\left[1 / \ln \left(r_{21}\right)\right]\left[\ln \left|\varepsilon_{32} / \varepsilon_{21}\right|+q(p)\right] \\
q(p)=\ln \left(\frac{r_{21}^{p}-s}{r_{32}^{p}-s}\right) \\
s=\operatorname{sign}\left(\varepsilon_{32} / \varepsilon_{21}\right)
\end{gathered}
$$

where $\varepsilon_{i j}=\phi_{j}-\phi_{i}$ and $\phi_{i}$ is the simulation value of the variable under interest on the $i$ th mesh. The above equations are solved with initial guess as $q=0$.

Step 4: The extrapolated values of the variables are calculated using Eq. (33):

$$
\phi_{\text {ext }}=\left(r_{21}^{p} \phi_{1}-\phi_{2}\right) /\left(r_{21}^{p}-1\right)
$$

Step 5: Approximate relative error in a dimensionless form is given by Eq. (34),

$$
e_{a}^{21}=\left|\frac{\phi_{1}-\phi_{2}}{\phi_{1}}\right|
$$

and the estimated extrapolated relative error is given by Eq. (35):

$$
e_{\mathrm{ext}}^{21}=\left|\frac{\phi_{\mathrm{ext}}^{21}-\phi_{1}}{\phi_{\mathrm{ext}}^{21}}\right|
$$

Finally the GCI is given by Eq. (36):

$$
\mathrm{GCI}_{\text {fine }}^{21}=\frac{F_{s} e_{a}^{21}}{r_{21}^{p}-1}
$$

$F_{s}$ is factor of safety and $F_{s}=1.25$ (ASME, 2009, 2-4.1). Using this value of $F_{s}$ would result in a GCI with $95 \%$ confidence interval. The variables under study, their extrapolated values, approximate errors, extrapolated errors, and GCI are given in Table 4.

As mentioned earlier, the uncertainty was calculated at only one point in time to give a first estimate of solution verification. Detailed uncertainty analysis is not in the scope of this work. As for vapor penetration, based on the analysis (at $1.4 \mathrm{~ms}$ ASOI) with observed order $p=0.6$, the value would be reported (for the finest grid of resolved orifice, $\phi_{1}$ ) as $52.38 \pm 1.85 \mathrm{~mm}$. Similarly the axial velocity and mixture fraction would be reported as $72.24 \pm 1.26 \mathrm{~m} / \mathrm{s}$ and $0.113 \pm 9.2 \times 10^{-4}$, respectively.

The plot of the key variables under consideration for the three grids is shown in Fig. 10. Liquid penetration could not be used for uncertainty analysis as the values for unresolved $(0.125 \mathrm{~mm})$ and resolved meshes were very close to each other. The simulations showed that there is a relatively clearer trend due to grid refinement for vapor penetration. However, liquid penetration is also shown here for reference.

The uncertainty quantified by GCI is plotted as an error bar for the finest grid and validated with experimental data (for the key variables) later in the results (Section 8).

The extrapolated value (represented by a blue dot at $t=1.4 \mathrm{~ms}$ ) for vapor penetration is a bit lower than the finest grid but it is seen that for axial velocity and mixture fraction, the extrapolated values are close to the values for the finest grid resolution used, which suggests that the finest grid resolution is reasonably sufficient for the purpose of this study. 
TABLE 4: Uncertainty analysis

\begin{tabular}{|c|c|c|c|c|}
\cline { 2 - 5 } \multicolumn{1}{c|}{} & $\begin{array}{c}\text { Vapor penetration } \\
(\mathbf{1 . 4} \mathbf{~ m s} \text { ASOI) }\end{array}$ & Vapor penetration $(\boldsymbol{p}=\mathbf{1})$ & $\left.\boldsymbol{U}_{\boldsymbol{a x}} \mathbf{( 2 5} \mathbf{~ m m}\right)$ & $\boldsymbol{Z} \mathbf{( 2 5} \mathbf{~ m m})$ \\
\hline $\boldsymbol{h}_{\mathbf{1}}$ & $28 \mu \mathrm{m}$ & $28 \mu \mathrm{m}$ & $28 \mu \mathrm{m}$ & $28 \mu \mathrm{m}$ \\
\hline $\boldsymbol{h}_{\mathbf{2}}$ & $125 \mu \mathrm{m}$ & $125 \mu \mathrm{m}$ & $125 \mu \mathrm{m}$ & $125 \mu \mathrm{m}$ \\
\hline $\boldsymbol{h}_{\mathbf{3}}$ & $250 \mu \mathrm{m}$ & $250 \mu \mathrm{m}$ & $250 \mu \mathrm{m}$ & $250 \mu \mathrm{m}$ \\
\hline $\boldsymbol{r}_{\mathbf{2 1}}$ & 4.46 & 4.46 & 4.46 & 4.46 \\
\hline $\boldsymbol{r}_{\mathbf{3 2}}$ & 2 & 2 & 2 & 2 \\
\hline $\boldsymbol{\phi}_{\mathbf{1}}$ & 52.38 & 52.38 & 72.24 & 0.113 \\
\hline $\boldsymbol{\Phi}_{\mathbf{2}}$ & 54.54 & 54.54 & 77.23 & 0.119 \\
\hline $\boldsymbol{\phi}_{\mathbf{3}}$ & 56.41 & 56.41 & 84.9 & 0.134 \\
\hline $\boldsymbol{p}$ & 0.6 & 1 & 1.19 & 1.55 \\
\hline $\boldsymbol{\phi}_{\mathbf{e x t}}^{\mathbf{2 1}}$ & 50.91 & 51.77 & 71.23 & 0.112 \\
\hline $\boldsymbol{e}_{\boldsymbol{a}}^{\mathbf{2 1}}$ & $4.11 \%$ & $4.11 \%$ & $6.91 \%$ & $5.94 \%$ \\
\hline $\boldsymbol{e}_{\mathbf{e x t}}^{\mathbf{2 1}}$ & $2.91 \%$ & $1.20 \%$ & $1.42 \%$ & $0.65 \%$ \\
\hline $\mathbf{G C I}_{\mathbf{f i n e}}^{\mathbf{2 1}}$ & $3.53 \%$ & $1.48 \%$ & $1.75 \%$ & $0.81 \%$ \\
\hline
\end{tabular}

\subsection{Comparison between Unresolved Cartesian and Cylindrical Core}

Referring again to Fig. 7, the unresolved mesh has a cartesian core and the resolved mesh has a cylindrical core. This work intends to use the cartesian core for unresolved mesh for all comparisons as this is what is typically used in contemporary spray simulations. However, a comparison is made between cartesian and cylindrical mesh for the same grid size around the orifice $(0.125 \mathrm{~mm})$ to highlight the influence of using of different cores on the results. The five-step procedure for uncertainity analysis is not used for the comparison of cartesian and cylindrical cores, because they are not geometrically similar grids and both of them have the same minimum grid size $(0.125 \mathrm{~mm})$. Therefore only the normalized relative difference (relDiff) of the values are presented in percentage in Table 5. These values are not necessarily error percentages but merely a difference because of the mesh topology used.

The plots of important variables under consideration are shown in Fig. 11. It is seen that liquid and vapor penetrations are relatively higher for the cylindrical core. One reason for this could be that there is more numerical diffusion for the cartesian core. For this same reason, the axial velocity and mixture fraction are also higher for the cylindrical core. It is also seen from Fig. 11(c) that the vapor is spread out to a small extent, more in the radial direction for the cartesian core, showing that there could be more diffusion. Also the cylindrical core is aligned to the spray to a greater extent, in all directions, whereas the cartesian core is aligned with the spray only at 90 degree intervals to the spray axis. A contour plot of temperature on the $x-y$ plane at $25 \mathrm{~mm}$ from the injector ( $z$-axis is the spray axis) is shown in Fig. 12.

It is seen from the contour plot that the cylindrical core has a clearer circular cross section of the spray. A detailed analysis of the differences between the cores is not in the scope of this study, but could be interesting for future work. Here the goal was to highlight the influence of choice of core (of the mesh) on the results. Following this, all discussion for unresolved mesh refers to the cartesian core.

Volume 31, Issue 4, 2021 
(a)
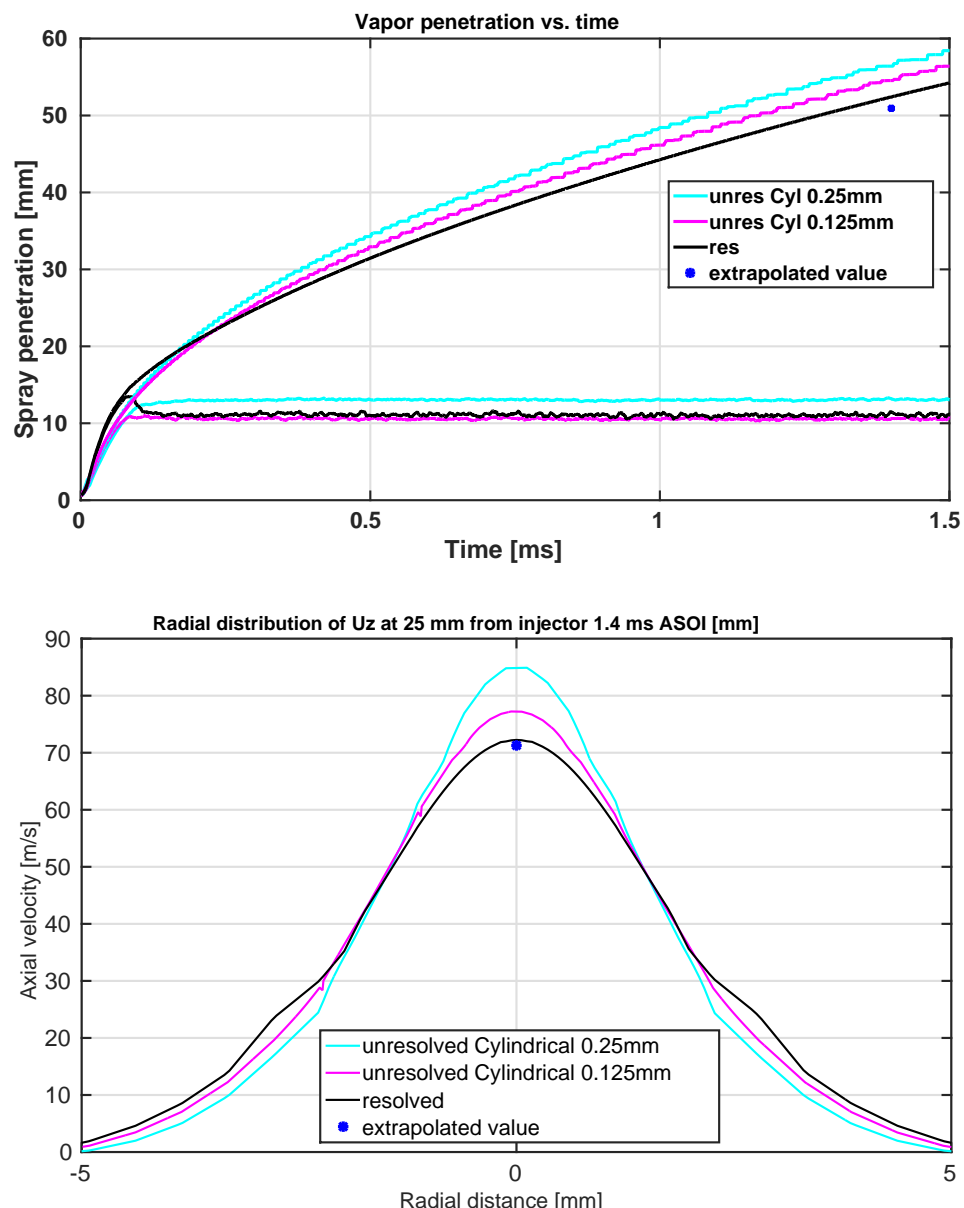

(b)

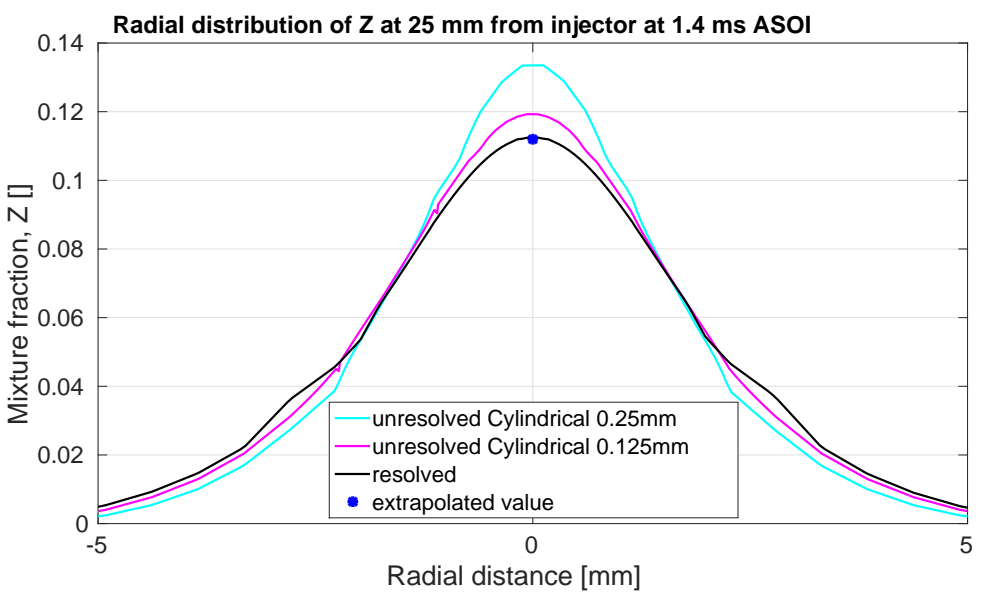

FIG. 10: Grid convergence of key variables. (a) Vapor penetration; radial distributions of (b) axial velocity and (c) mixture fraction. 
TABLE 5: Comparison of cartesian and cylindrical cores

\begin{tabular}{|l|c|c|c|c|}
\cline { 2 - 5 } \multicolumn{1}{c|}{} & $\begin{array}{c}\text { Vapor penetration } \\
(\mathbf{1 . 4} \mathbf{~ m s} \text { ASOI) }\end{array}$ & Vapor penetration & $\left.\boldsymbol{U}_{\boldsymbol{a x}} \mathbf{( 2 5} \mathbf{~ m m}\right)$ & $\boldsymbol{Z} \mathbf{( 2 5} \mathbf{~ m m})$ \\
\hline $\begin{array}{l}\text { Cartesian core, min } \\
\text { grid size }\end{array}$ & $28 \mu \mathrm{m}$ & $28 \mu \mathrm{m}$ & $28 \mu \mathrm{m}$ & $28 \mu \mathrm{m}$ \\
\hline $\begin{array}{l}\text { Cylindrical core, } \\
\text { min grid size }\end{array}$ & $125 \mu \mathrm{m}$ & $125 \mu \mathrm{m}$ & $125 \mu \mathrm{m}$ & $125 \mu \mathrm{m}$ \\
\hline $\boldsymbol{\phi}_{\mathbf{1}}$ (cartesian core) & 51.27 & 8.69 & 69.67 & 0.107 \\
\hline $\begin{array}{l}\boldsymbol{\phi}_{\mathbf{2}} \text { (cylindrical } \\
\text { core) }\end{array}$ & 54.54 & 10.61 & 77.23 & 0.119 \\
\hline $\begin{array}{l}\text { relDiff (with regard } \\
\text { to cartesian core) }\end{array}$ & $6.38 \%$ & $22.18 \%$ & $10.88 \%$ & $11 \%$ \\
\hline
\end{tabular}

\section{RESULTS}

Results are presented first for influence of resolving injector orifice followed by influence of considering stripped off droplets. The significance of including the bubble is also discussed towards the end.

\subsection{Influence of Resolving the Injector Orifice}

In order to assess the influence of resolving the injector orifice on spray formation, the following parameters are studied: liquid and vapor penetration, evaporation rate, mixing of fuel with air, velocity fields, and turbulence.

\subsubsection{Liquid and Vapor Penetration}

Liquid penetration was defined as the maximum distance from the injector containing $99 \%$ of the injected liquid mass. Vapor penetration was defined as the maximum distance from the injector containing at least $0.1 \%$ fuel vapor mass fraction. A plot of liquid and vapor penetration for the resolved and unresolved orifice meshes is shown in Fig. 13.

The values of liquid and vapor penetration for the resolved mesh are higher than for the unresolved mesh. The reason for the differences in liquid and vapor penetration can be explained by comparing the evaporation rates for the two meshes.

\subsubsection{Evaporation Rate}

The evaporation rate was defined as the ratio of evaporated liquid mass to injected liquid mass and is plotted for both meshes against time in Fig. 14.

We can see that the evaporation rate for the resolved mesh is lower. One possible reason for this is because the gas in the smaller grid cells becomes saturated relatively quicker than the larger grid cells in the unresolved mesh. Therefore the evaporation rate is lower and liquid penetration is higher for the resolved mesh. Another possible reason is that, as explained in Section 5.1, there is more numerical diffusion in the radial direction for the unresolved mesh due to the cartesian core. As seen for velocity and mixture fraction, a similar trend for gas temperature is expected. This diffusion would then reduce the peak temperature in the core of 
(a)
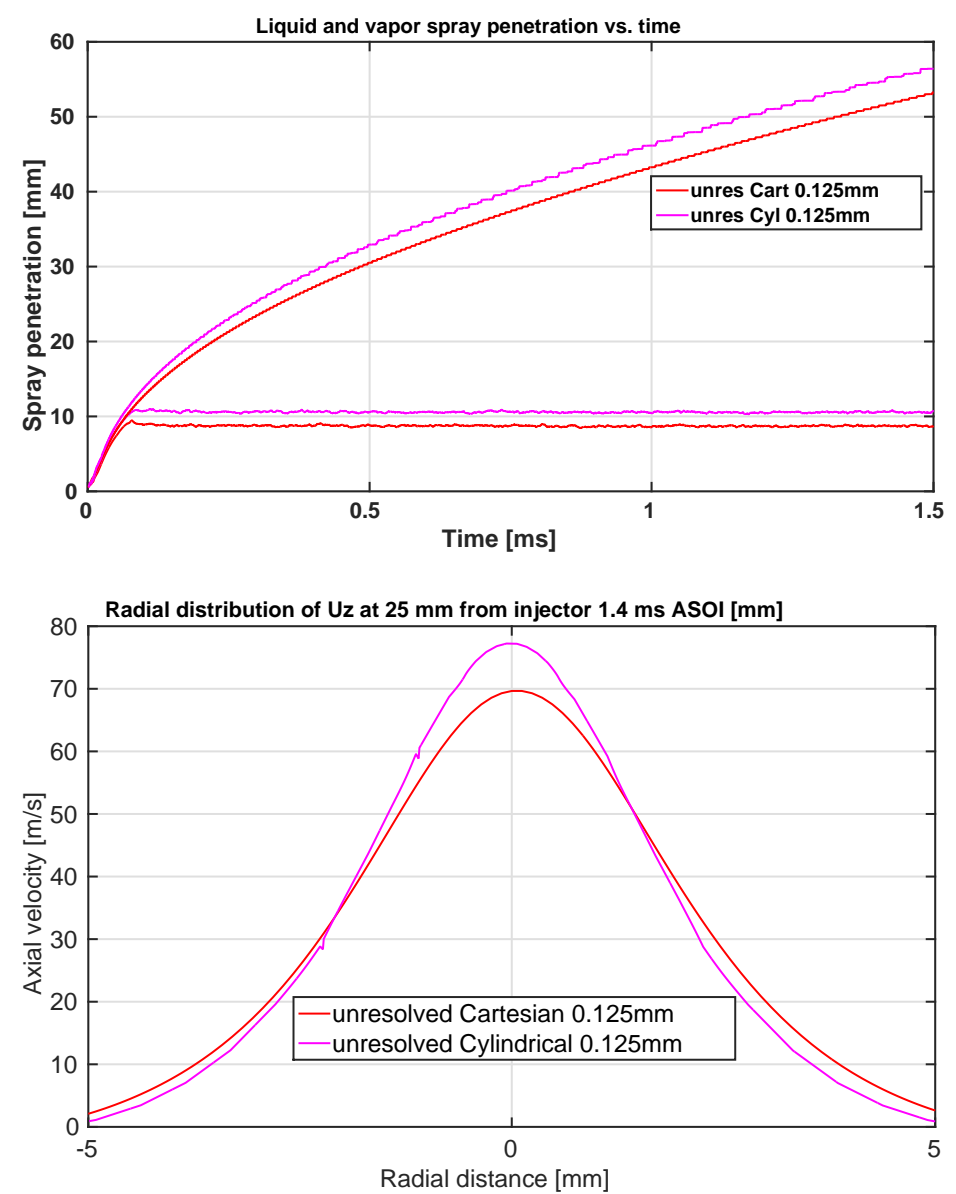

(b)

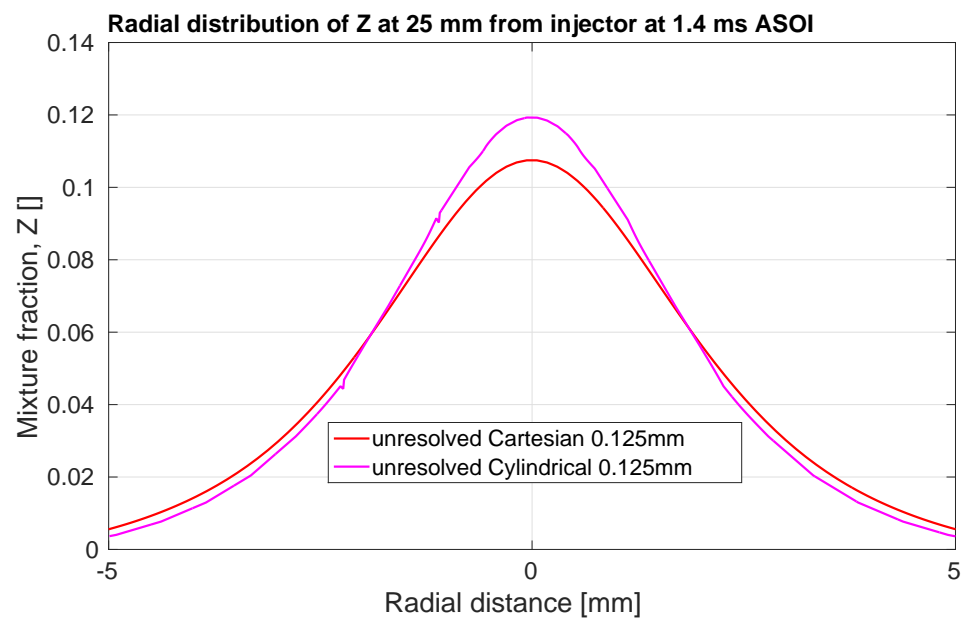

FIG. 11: Plot of key variables for cartesian and cylindrical cores. (a) Vapor penetration; radial distributions of (b) axial velocity and (c) mixture fraction. 


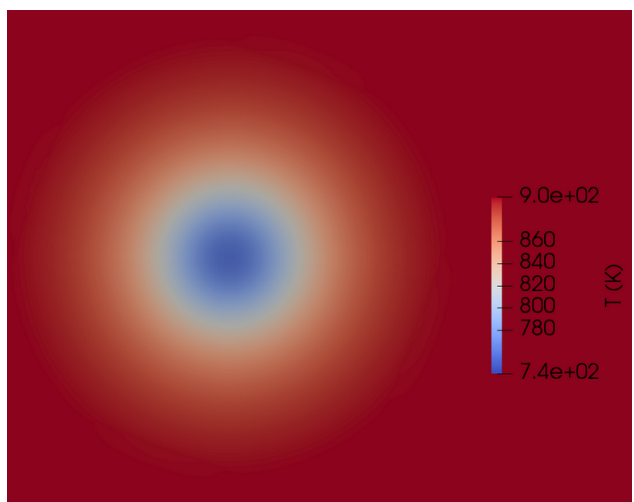

(a)

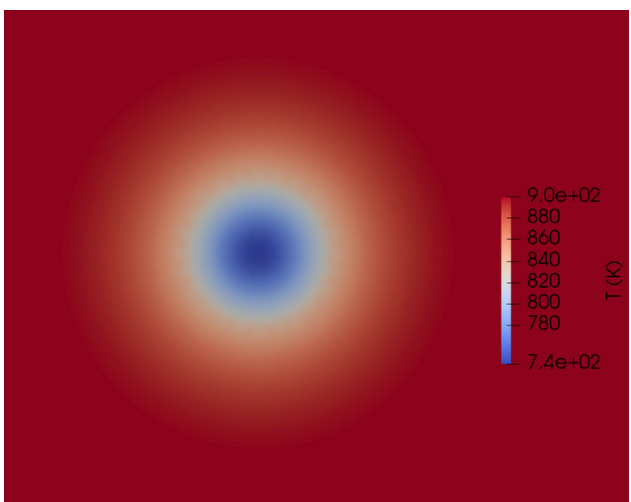

(b)

FIG. 12: Comparison between (a) cartesian and (b) cylindrical core ( $\min$ grid size $=0.125 \mathrm{~mm}$ )

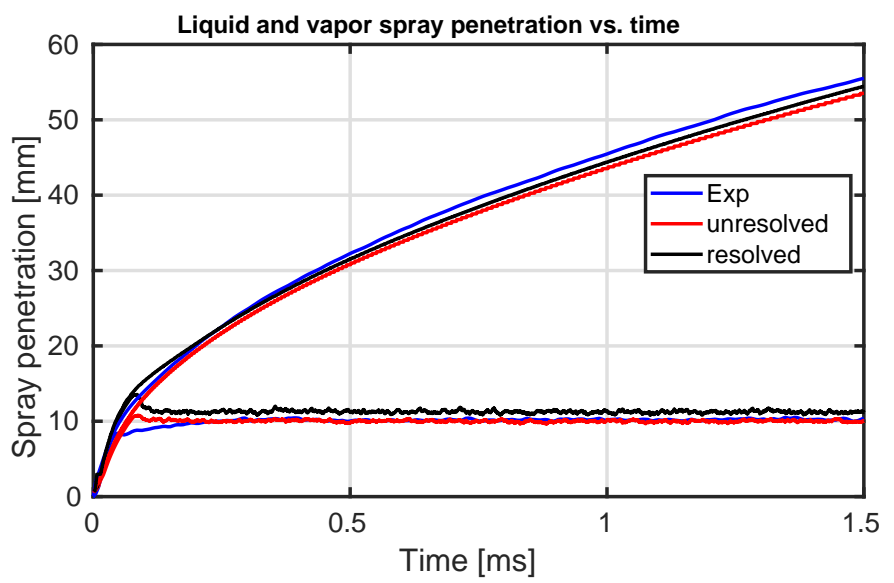

FIG. 13: Liquid and vapor penetration comparison

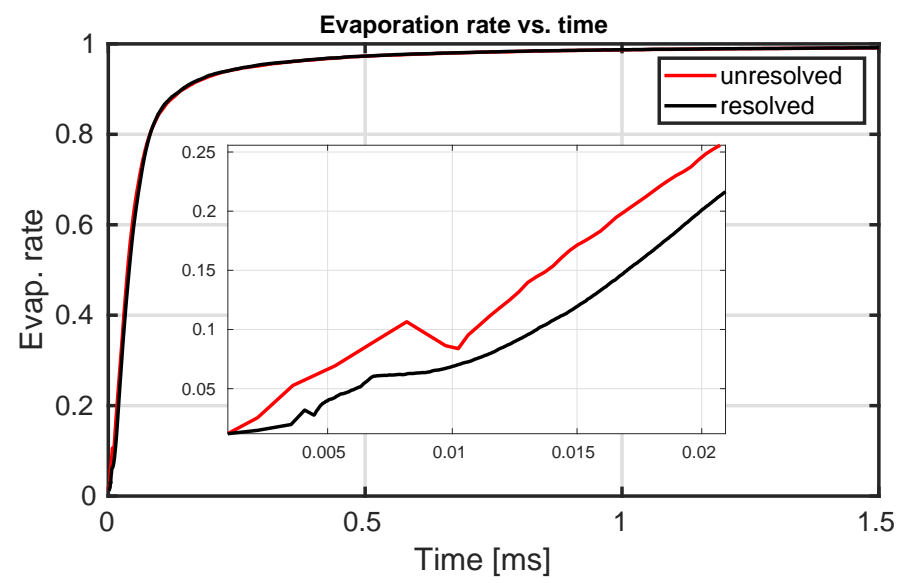

FIG. 14: Evaporation rate comparison 
the spray for the unresolved mesh and disperse it more in the radial direction, which would result in reduced evaporation around the core.

\subsubsection{Prediction of the Mixing Field}

An important measure of the validity of a spray model is the accuracy of prediction of mixing fuel with air. Plots of the axial and radial mixture fraction profiles are shown in Fig. 15.

Both the unresolved and resolved meshes showed a reasonably good prediction of the mixture fraction when compared to experimental data, especially for the axial profile. However, the axial profiles for the two meshes differed close to the injector. Experimental data was not available for the near nozzle region, therefore it was not possible to validate the predictions in this region. One reason for higher axial mixture fraction of resolved mesh close to the injector is due to the smaller grid cells, which become saturated more quickly than in the unresolved mesh; another reason is that there is more diffusion in the radial direction for the unresolved mesh [a more radially dispersed spray was seen for the cartesian core in Fig. 11(c)].

Regarding the radial mixture fraction, there was a reasonable agreement with the experimental data, although there is a difference in peak values. The peak for the resolved mesh is slightly higher and closer to the experiment for the same reasons mentioned earlier.

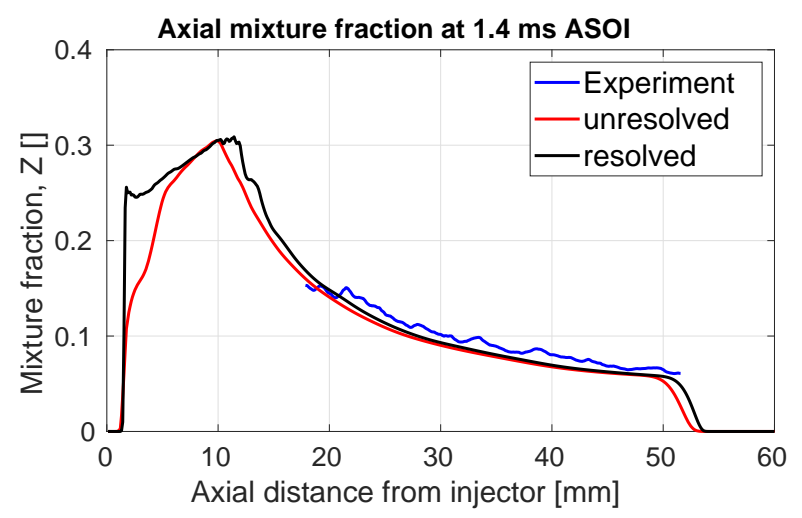

(a)

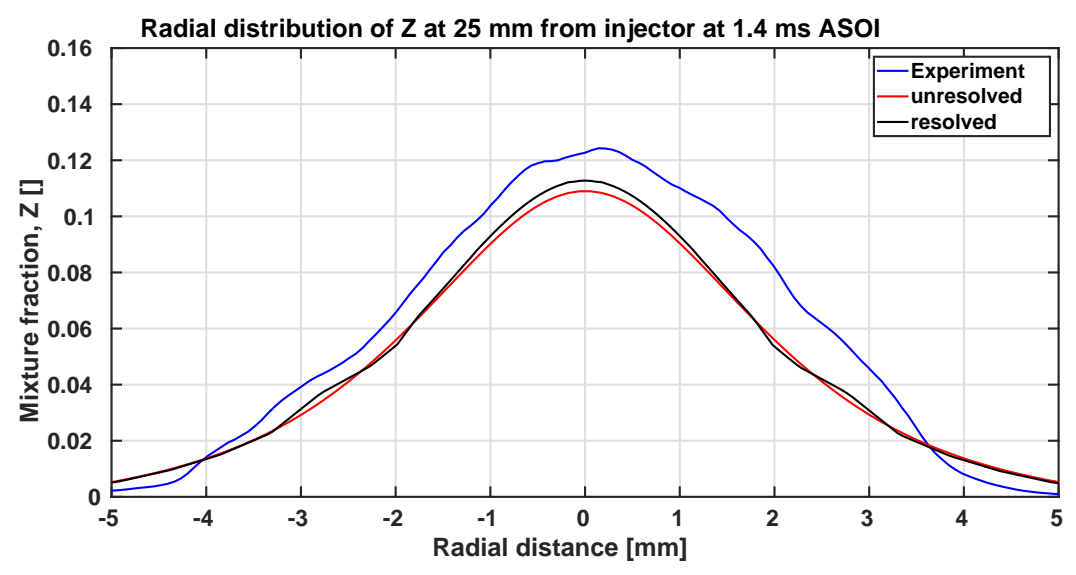

FIG. 15: Mixture fraction profiles at $1.4 \mathrm{~ms}$ ASOI. (a) Axial and (b) radial profiles. 


\subsubsection{Prediction of the Velocity Field}

The radial and axial distribution of the axial velocity for both resolved and unresolved meshes are plotted against experimental data in Fig. 16. Note that the experimental data in the ECN database provided by Sandia did not have velocity measurements. Therefore experimental data provided by IFPEN (IFPEN, 2020) for the same ECN spray A boundary conditions were taken. Once again, no experimental data was close to the injector. This made it difficult to validate the simulation data in the near nozzle region. However, further away from the injector $(>20 \mathrm{~mm})$, there was a good agreement between the simulated and experimental data. Differences between the two meshes were mainly seen close to the injector. In particular, the resolved mesh showed a higher velocity because momentum transfer from the liquid to the smaller mass of gas in the finer grid cells of the resolved mesh resulted in a higher velocity. Another possible reason as mentioned earlier is that there is more diffusion of velocity in the radial direction for the unresolved mesh with the cartesian core [as seen in Fig. 11(b)], resulting in a lower velocity. The radial velocity showed a reasonable agreement with the experimental data.

\subsubsection{Turbulence and Mixing Rate}

Accurate prediction of the turbulent kinetic energy $(k)$ and dissipation $(\epsilon)$ values depends on how well the velocity gradients are resolved. Plots of the maximum values of $k$ and $\epsilon$ versus

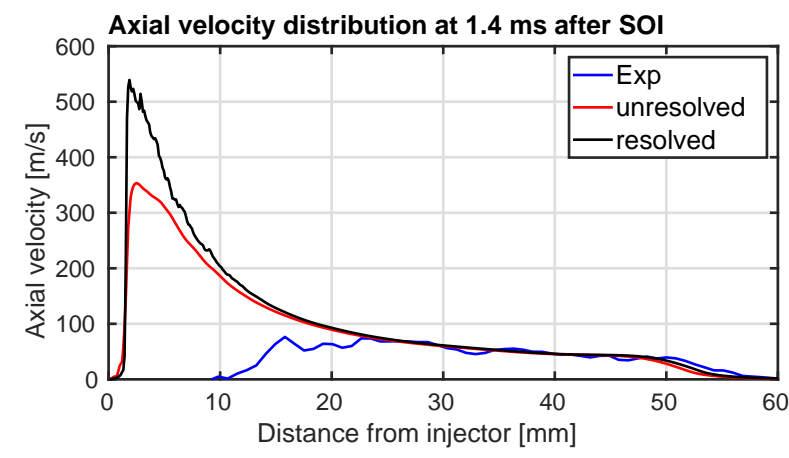

(a)

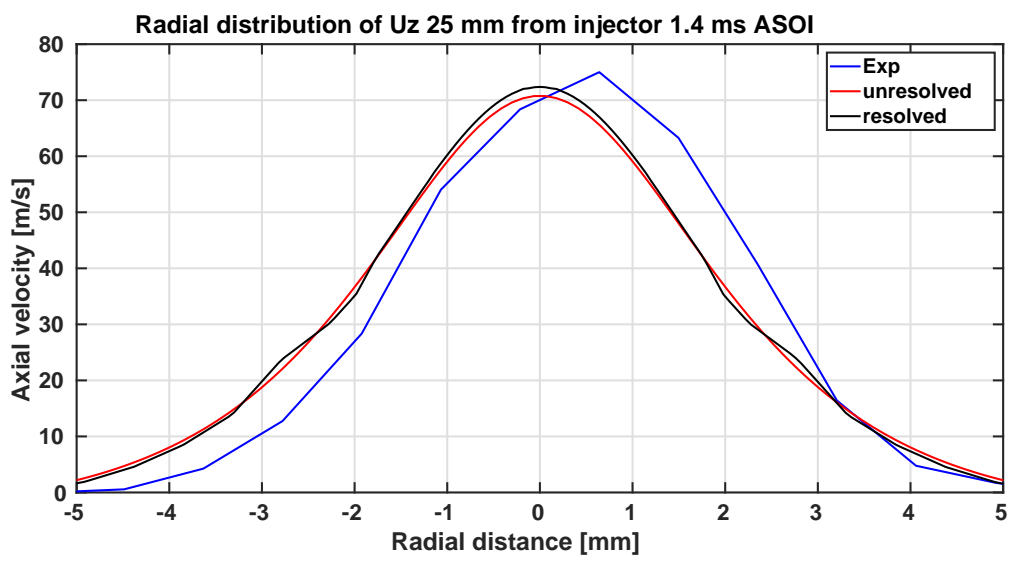

FIG. 16: Velocity profiles at $1.4 \mathrm{~ms}$ ASOI. (a) Axial and (b) radial profiles.

Volume 31, Issue 4, 2021 
time for the two meshes are shown in Fig. 17. Both $k_{\max }$ and $\epsilon_{\max }$ were relatively higher for the resolved mesh. This was because the velocity gradients were also higher for the resolved mesh owing to the higher gas velocity and smaller grid cell size. Based on $k$ and $\epsilon$, mass weighted average of the mixing rate for the fuel with air was calculated from Eq. (37):

$$
\text { MixRate }=\frac{\sum_{i=11}^{n_{\text {cells }}} \rho_{i} V_{i}\left(\epsilon_{i} / k_{i}\right)}{\sum_{i=1}^{n_{\text {cells }}} \rho_{i} V_{i}}
$$

The mixing rate is useful for predicting combustion of fuel. A higher mixing rate gives rise to a higher rate of reaction. The mass weighted average of the mixing rate of the two simulation cases for stoichiometric and rich region is shown in Fig. 18. As expected, the resolved case had a higher mixing rate for both the stoichiometric and rich regions.

\subsubsection{Influence of Asymmetric Velocity Field}

One question to be answered was how well the two simulation meshes would predict a spray with an asymmetrical injection velocity field. The procedure of creating an asymmetrical velocity field in the simulations is described.

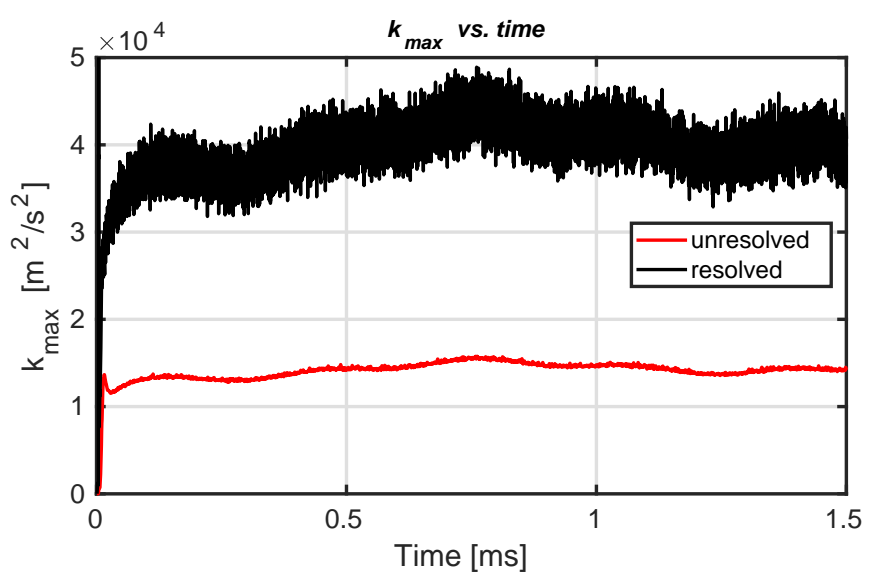

(a)

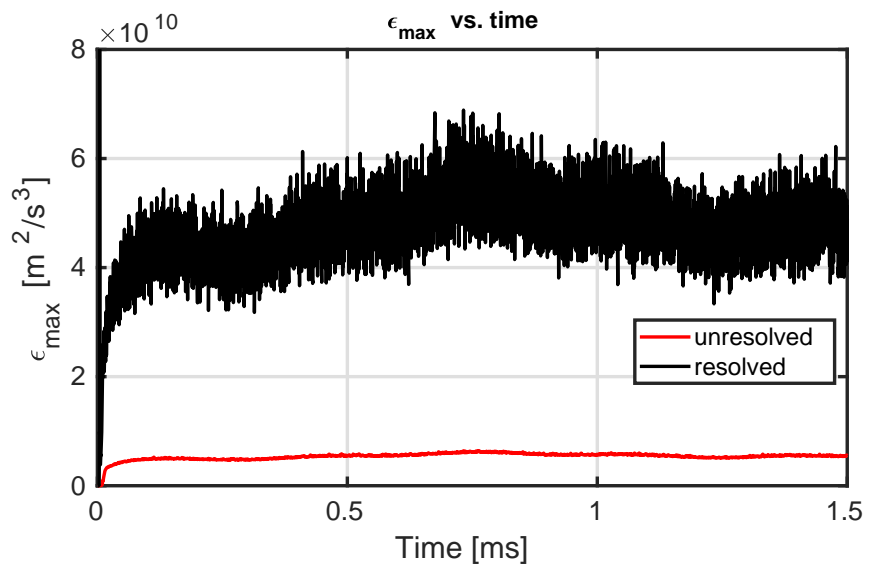

FIG. 17: Maximum turbulent (a) kinetic energy $\left(k_{\max }\right)$ and (b) dissipation $\left(\epsilon_{\max }\right)$ vs. time 


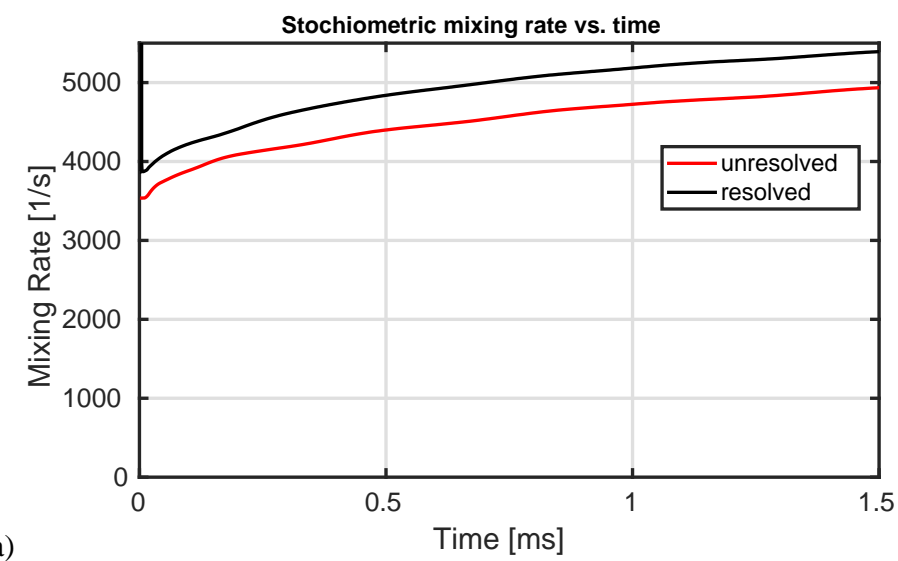

(a)

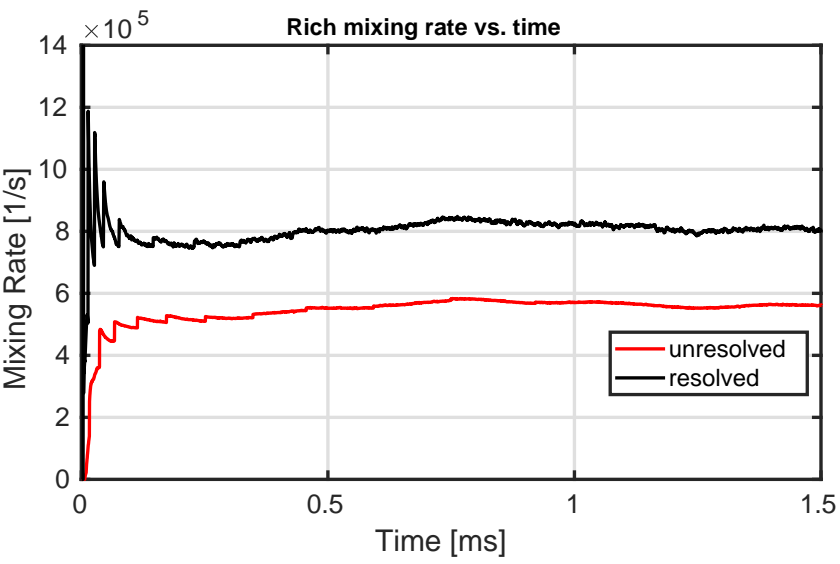

FIG. 18: (a) Stochiometric and (b) rich mixing rate vs. time

The droplet velocity vector is given by Eq. (38),

$$
\overrightarrow{U_{d}}=U_{i} \hat{i}
$$

where $U_{i}$ is the magnitude of the injection velocity that can be calculated from the mass flow rate for any given time, and $\hat{i}$ is the unit direction vector given by Eq. (39),

$$
\hat{i}=\cos \theta_{s} \vec{i}_{\text {in }}+\vec{n}
$$

where $\theta_{s}$ is randomly chosen to have a value between the inner and outer spray angles (given as inputs), $\vec{i}_{\text {in }}$ is the input direction vector (along the negative $z$-direction for both simulation cases), and $\vec{n}$ is vector normal to the plane containing spray axis ( $\vec{n}$ is in the $x-y$ plane) given by Eq. (40),

$$
\vec{n}=\sin \theta_{s}\left\{\overrightarrow{C_{1}} \cos \beta+\overrightarrow{C_{2}} \sin \beta\right\}
$$

where $\overrightarrow{C_{1}}$ and $\overrightarrow{C_{2}}$ are tangential input vectors that are orthogonal to each other and also orthogonal to the input injection direction, and $\beta$ is the angle that controls the orientation of the spray on the $x-y$ plane.

Volume 31, Issue 4, 2021 
An asymmetrical spray was generated for both simulation cases. This is achieved by changing the angle $\beta$ such that $0<\beta<\pi$ (the convention in OpenFOAM is such that $\beta=0$ is along the positive $y$-axis and increases in a clock wise direction). Thereby $\vec{n}$ was limited to the righthand half of the $x-y$ plane (where $x$ is always positive), resulting in an asymmetrical spray. To exaggerate the asymmetry so that it was clearly visible, the outer spray angle is increased to $15^{\circ}$ (inner spray angle is $6^{\circ}$ ). The resulting spray (colored by droplet mass) for the unresolved mesh, is shown in Fig. 19 (the spray is seen in the direction of the $y$-axis).

Contour plots of gas temperature for both meshes are shown in Fig. 20. For the resolved orifice, perturbations were detected at the leading edge of the spray and it was slightly asymmetric. These perturbations were absent for the unresolved case, which exhibited a smoother and relatively symmetrical temperature field.

Contour plots of the gas velocity are shown in Fig. 21. Differences in gas velocity are small, and finer differences can be seen around the leading edge of the spray. The plot of mixture fraction is shown in Fig. 22. Here a bigger difference can be seen at the leading edge of the spray. As in the case of temperature, fine and slightly asymmetric perturbations can be seen at the leading edge of the spray for the resolved mesh but not for the unresolved mesh.

From the results, it can be concluded that local patterns induced at the leading edge of the spray due to an asymmetric velocity profile are not captured in the unresolved mesh. It should be noted that these differences can also arise out of the difference in meshes used for the two cases.

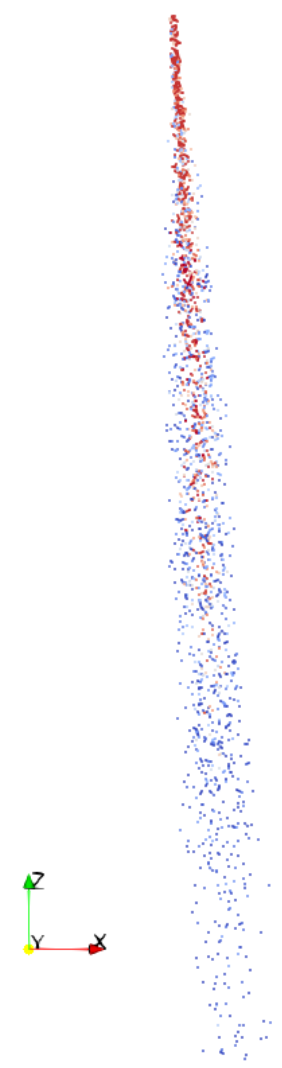

FIG. 19: Lagrangian spray at $0.1 \mathrm{~ms}$ ASOI 


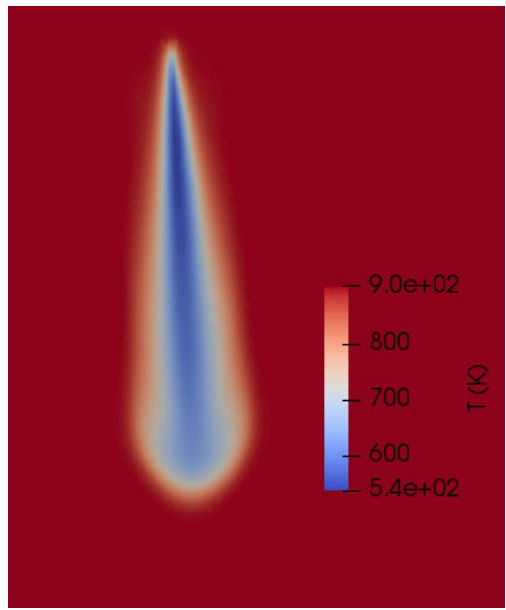

(a)

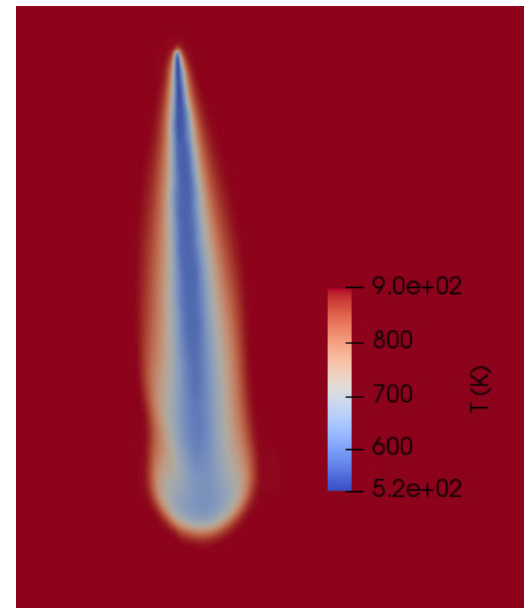

(b)

FIG. 20: Contour plots of temperature at $0.1 \mathrm{~ms}$ ASOI. (a) Unresolved and (b) resolved injector orifice.

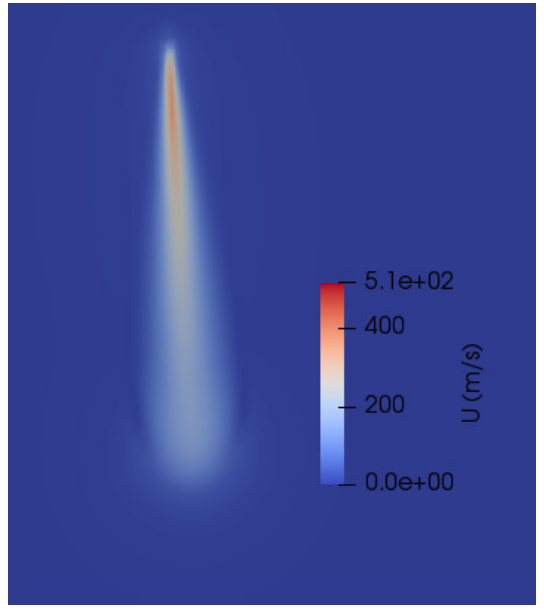

(a)

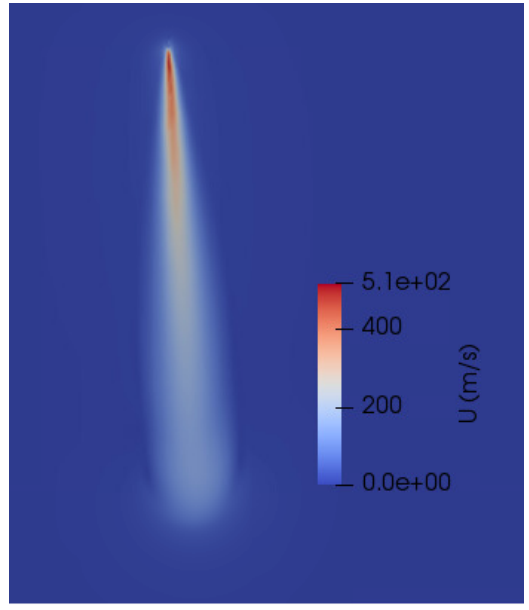

(b)

FIG. 21: Contour plots of velocity at $0.1 \mathrm{~ms}$ ASOI. (a) Unresolved and (b) resolved injector orifice.

\subsection{Influence of Creating New Child Blobs from Stripped Off Mass}

The stripped off mass is defined as the liquid mass that is removed by secondary breakup (Section 2.3). Two simulation cases were studied for the same ECN spray A conditions mentioned in Table 3: one case where the stripped off mass was used to create new child blob (if it was greater than $1 \%$ of the mass of the original parent blob) and the other where the stripped off mass was not used to create new child blob but was rather retained as one of the mass packages in the original blob (each blob is divided into 10 mass packages; see Section 2.2). The reason for studying this is as follows. The smaller stripped off droplets in the liquid blob transfer the same momentum as the bigger droplets. Creating new blobs from stripped off droplets is accurate in terms of momentum transfer. Thus we wanted to determine how much of an influence this will

Volume 31, Issue 4, 2021 


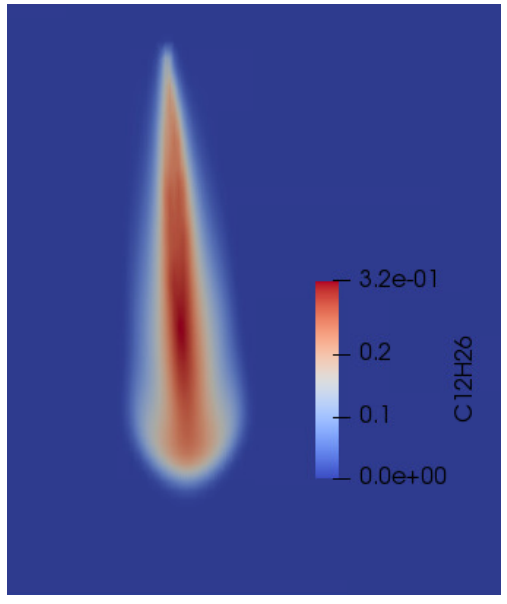

(a)

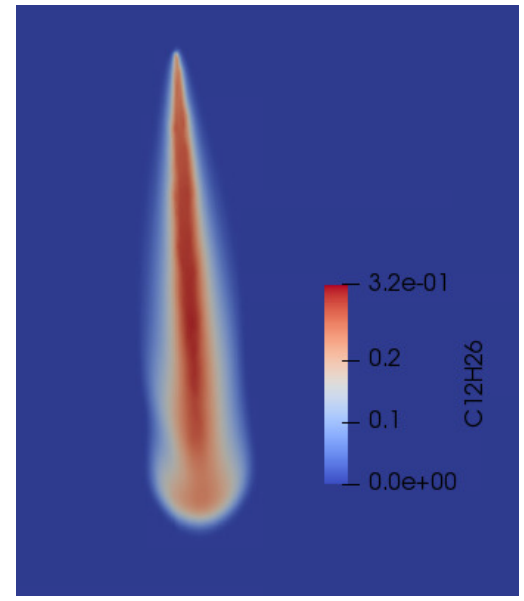

(b)

FIG. 22: Contour plots of mixture fraction at $0.1 \mathrm{~ms}$ ASOI. (a) Unresolved and (b) resolved injector orifice.

have under high pressure and temperature conditions of diesel sprays. The two simulation cases (stripped off and unstripped off) were compared with respect to liquid and vapor penetration, Sauter mean diameter (SMD), and velocity and mixture fraction fields of the gas phase. The comparison is performed for both unresolved and resolved mesh.

\subsubsection{Unresolved Mesh}

\subsubsection{Liquid and Vapor Penetration}

Liquid and vapor penetration were defined in the same way as in Section 6.1.1. The plot of liquid and vapor penetration for stripped off and unstripped off cases is shown in Fig. 23. It can be seen that the stripped off case predicted a slightly lower liquid penetration than the unstripped off case. However there is virtually no difference in the vapor penetration between the two simulation cases. To explain the difference in liquid penetration, it is useful to consider evaporation rate.

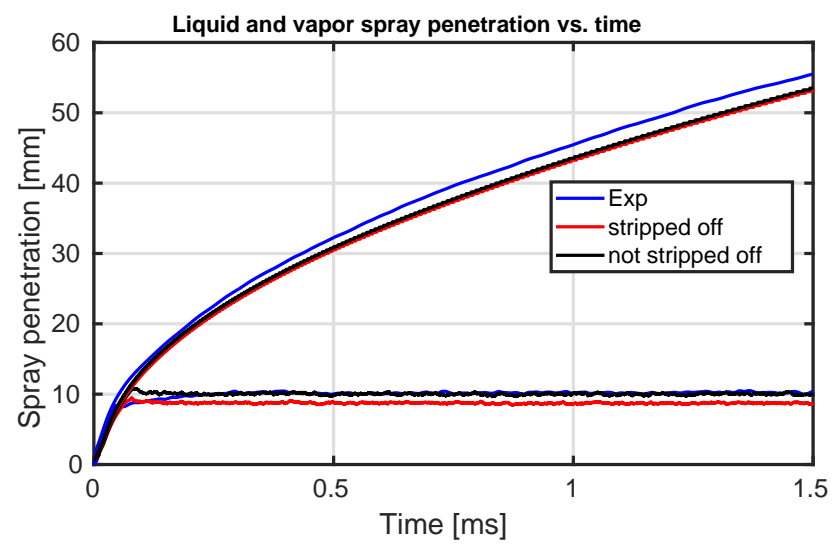

FIG. 23: Liquid and vapor penetration for unresolved orifice mesh 


\subsubsection{Evaporation Rate}

Evaporation rate is compared for the stripped off and unstripped off cases (Fig. 24). It can be seen that there is a difference in the evaporations rates, and the stripped off case has a slightly higher value. The reason for the difference in evaporation rates can in turn be explained with SMD, as given by Eq. (41),

$$
\mathrm{SMD}=\frac{\sum_{i=1}^{N_{\text {par }}} \sum_{j=1}^{n_{\text {blob }}} m_{j} D_{j}^{3}}{\sum_{i=1}^{N_{\text {par }}} \sum_{j=1}^{n_{\text {blob }}} m_{j} D_{j}^{2}}
$$

where $i$ refers to a specific parcel (blob) and $j$ refers to a specific size interval (or mass package) in the $i$ th parcel (blob), $N_{\text {par }}$ is the number of parcels (blobs), $n_{\text {blob }}$ is the number of size intervals in a blob, $m_{j}$ is the mass of droplets in a given mass package (the mass that corresponds to a given size interval), and $D_{j}$ is the diameter of the droplets in a given size interval. A plot of SMD versus time for the two cases is shown in Fig. 25. SMD of the stripped off case was found to be lower than the unstripped off case, suggesting that the blobs in the stripped off case have a larger surface area per unit volume, and thus evaporated slightly faster than the unstripped off case.

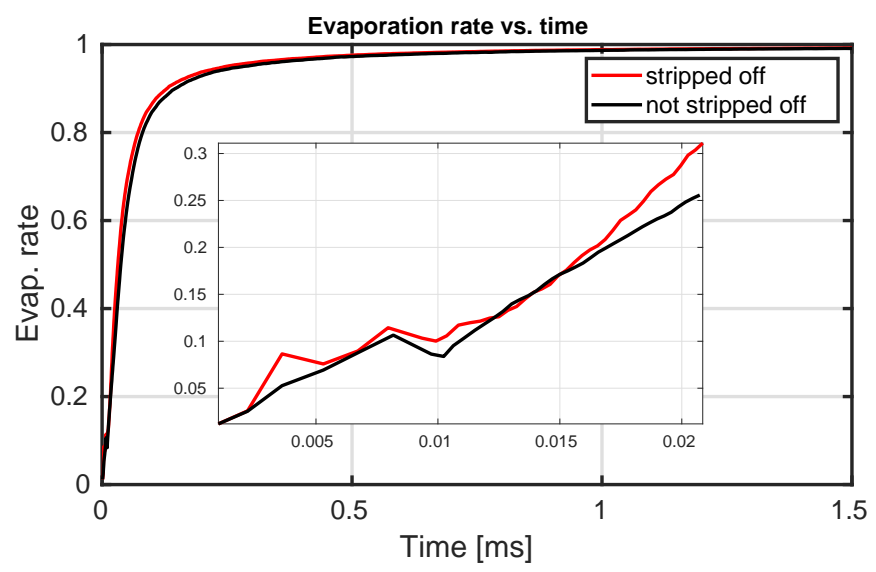

FIG. 24: Evaporation rate for unresolved orifice mesh

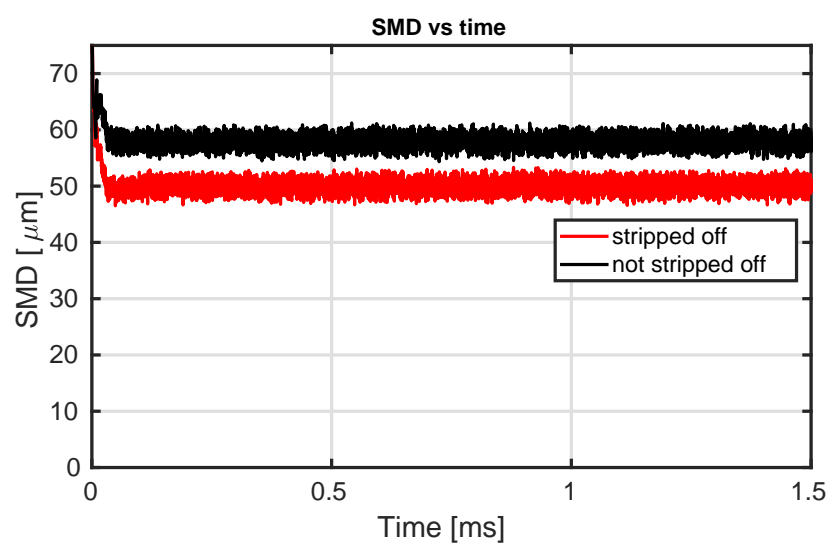

FIG. 25: SMD for the unresolved orifice mesh

Volume 31, Issue 4, 2021 
Since SMD does not reveal much about the mass of droplets, representations of the Lagrangian spray colored according to the droplet mass are shown in Fig. 26.

The stripped off case produced a lot of smaller droplets, confirmed by the glyph representation of the spray, sized by blob mass, shown in Fig. 27.

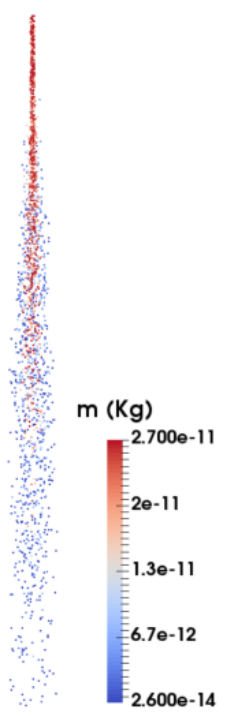

(a)

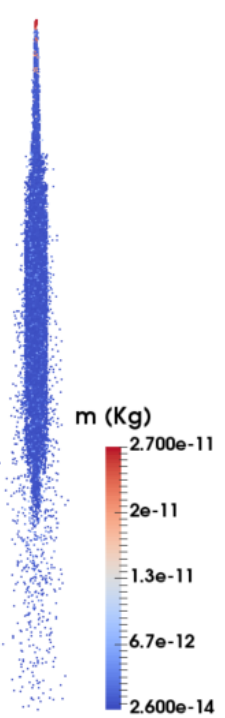

(b)

FIG. 26: Lagrangian spray at $0.1 \mathrm{~ms}$ ASOI for the unresolved mesh colored according to droplet mass. (a) Unstripped and (b) stripped off cases.

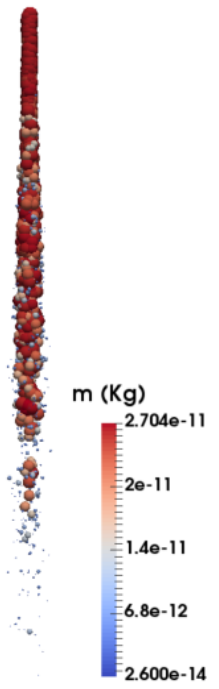

(a)

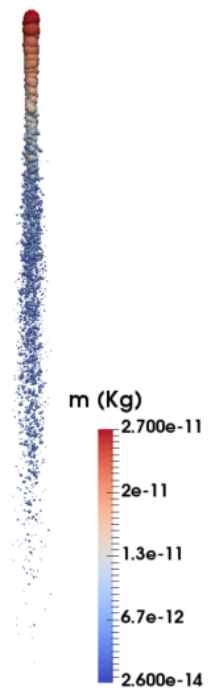

(b)

FIG. 27: Glyph representation of spray at $0.1 \mathrm{~ms}$ ASOI for the unresolved mesh sized according to blob mass. (a) Unstripped and (b) stripped off cases. 
Note that the difference in the liquid mass in the system (domain) is small between the two cases, with the unstripped off case having higher liquid mass due to the lower evaporation rate, as seen in Fig. 28.

The smaller droplets in the stripped off case also heated up faster as Fig. 29 shows (the temperature in the figure has been rescaled from $500-580 \mathrm{~K}$ to emphasize the difference in maximum values). The smaller and relatively hotter droplets of the stripped off case evaporated faster than those of the unstripped off case, leading to a lower liquid penetration.

\subsubsection{Resolved Mesh}

As for the unresolved orifice, two simulation cases were compared for the resolved injector orifice, one in which the stripped off droplets were used to create new blobs and the other in which

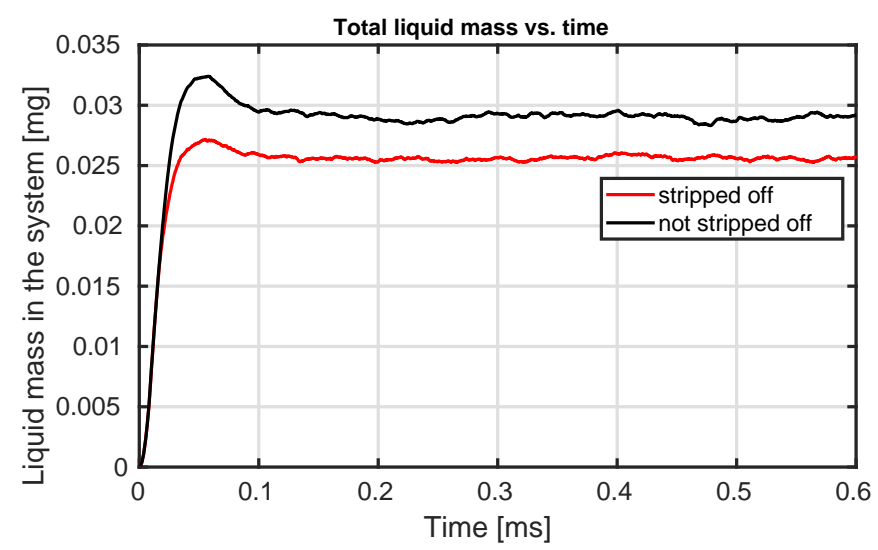

FIG. 28: Liquid mass in the system for the unresolved orifice mesh

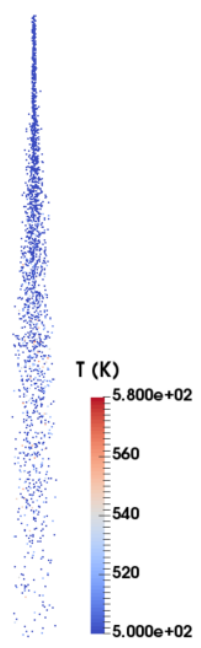

(a)

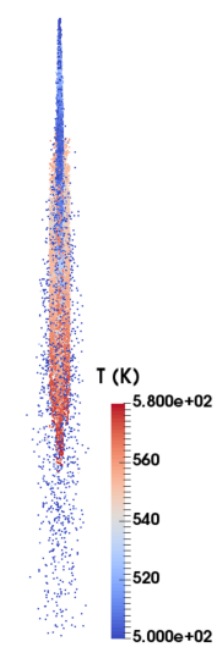

(b)

FIG. 29: Lagrangian spray at $0.1 \mathrm{~ms}$ ASOI for the unresolved mesh coloured according to droplet temperature. (a) Unstripped and (b) stripped off cases.

Volume 31, Issue 4, 2021 
they were retained in old blobs. A plot of the liquid and vapor penetration for the two cases is shown in Fig. 30. This plot shows that differences in liquid and vapor penetrations of the two cases were not significant. Figure 31 shows that differences in SMD were also not significant. The reason for insignificant difference can be explained by referring again to the earlier discussion on the difference between resolved and unresolved meshes. As discussed earlier in Section 6.1.4, the axial gas velocity was much higher for the resolved orifice, owing to the ease of transfer of momentum from liquid to gas in smaller grid cells of the resolved orifice case. The higher gas velocity caused a lower relative velocity between the liquid and gas and lower secondary breakup, resulting in fewer stripped off droplets for the resolved orifice. Since the number of stripped off droplets was relatively lower, they had less influence on the spray.

Representations of the Lagrangian spray colored according to the droplet mass are shown in Fig. 32. The glyph representations of the spray colored by droplet mass and the Lagrangian spray colored by droplet temperature are shown in Figs. 33 and 34, respectively.

Figures 32-34 shows that the stripped off case has produced relatively more, smaller droplets that are hotter than the unstripped off case. However, when compared to the same plots for the unresolved mesh (especially comparing the plot showing glyph of droplets sized by mass for the

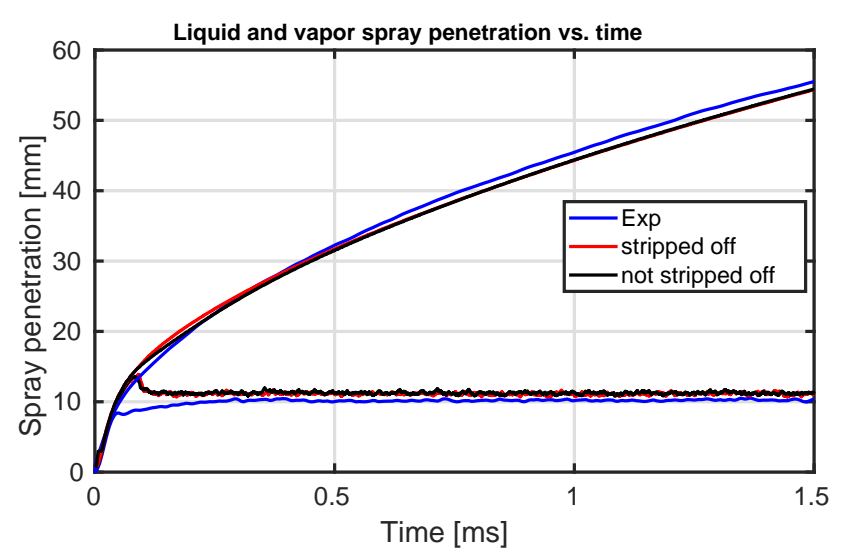

FIG. 30: Liquid and vapor penetration for the resolved orifice case

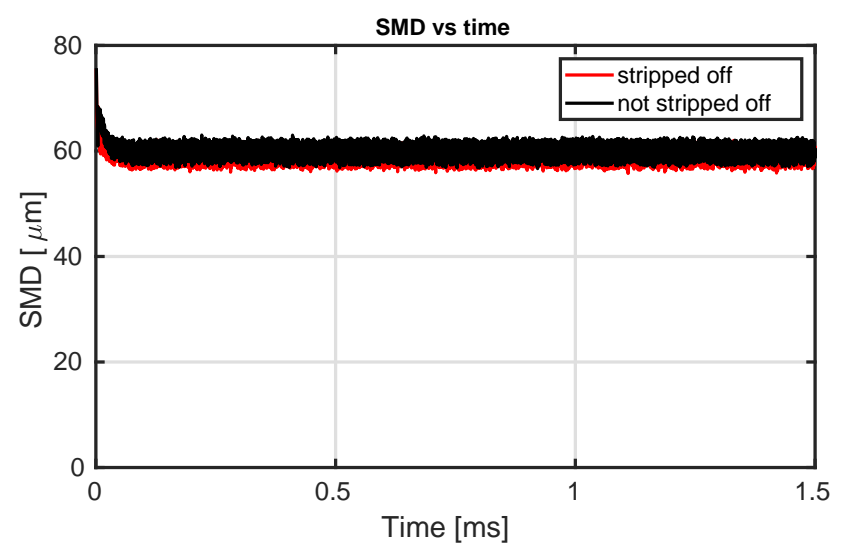

FIG. 31: SMD for the resolved orifice 


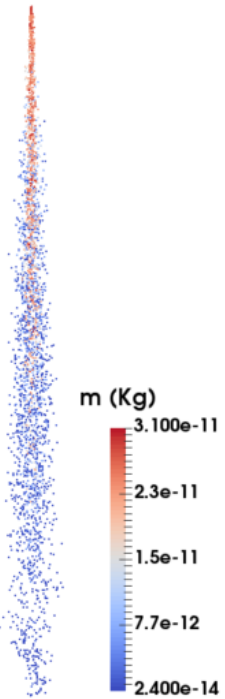

(a)

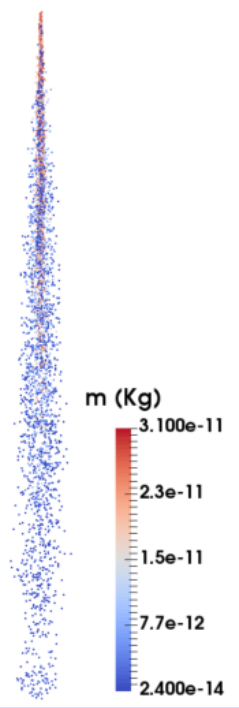

(b)

FIG. 32: Lagrangian spray at $0.1 \mathrm{~ms}$ ASOI for the resolved mesh colored according to droplet mass. (a) Unstripped and (b) stripped off cases.

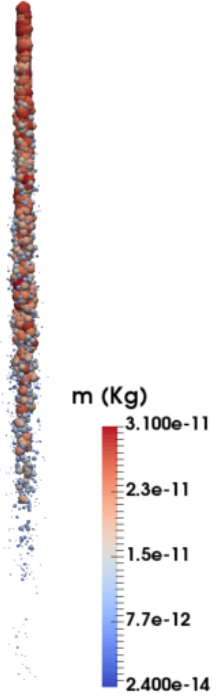

(a)

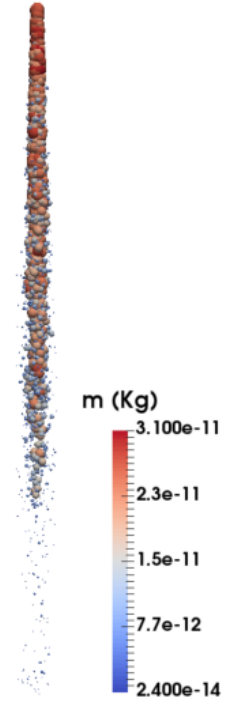

(b)

FIG. 33: Glyph representation of spray at $0.1 \mathrm{~ms}$ ASOI for the resolved mesh sized according to blob mass. (a) Unstripped and (b) stripped off cases.

stripped off case Figs. 27 and 33), it can be seen that the there are more, smaller stripped off droplets produced for the unresolved mesh. The influence of stripping off and producing smaller droplets is therefore relatively lower for the resolved mesh.

Volume 31, Issue 4, 2021 


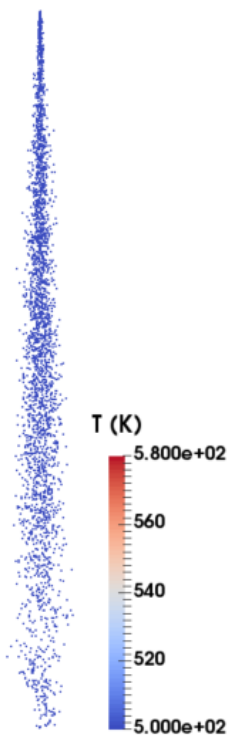

(a)

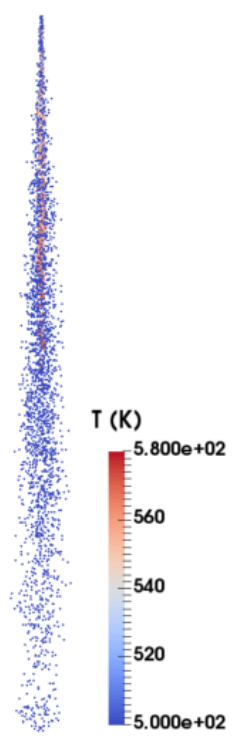

(b)

FIG. 34: Lagrangian spray at $0.1 \mathrm{~ms}$ ASOI for the resolved mesh colored according to droplet temperature. (a) Unstripped and (b) stripped off cases.

\subsubsection{Velocity and Mixture Fraction Profile}

Apart from measuring liquid and vapor penetration and droplet SMD, interaction of the spray with the surrounding air was also examined for both the resolved and unresolved injector orifice. The mixture fraction and velocity of the gas phase versus radial distance, for the stripped off and unstripped off cases, are plotted in Fig. 35.

It can be seen that as far as the mixing and velocity fields are concerned, the differences between the stripped off and unstripped off cases were insignificant for both the resolved and unresolved injector orifice. Thus the results overall suggest that under high pressure and temperature diesel engine-like operating conditions, the formation of new blobs from stripped off blob mass has an insignificant effect on the spray predictions. This could be because of the fact that under the high temperature evaporating conditions, the evaporation rate is high and therefore the smaller stripped off droplets are quickly evaporated.

\section{INFLUENCE OF BUBBLE}

The concept of bubble was discussed in Section 2.9. Here the importance of bubble is shown through the results of simulations for the same ECN spray A case. Bubble is used to reduce the dependency on grid cells, especially when larger grid cells are used. Here the discussion is based on simulations for the unresolved mesh where we can expect a stronger influence of bubble owing to coarser grid cells. The effect of including bubble is similar for both the stripped off and unstripped off cases. The focus in this section is limited to the stripped off case, where we can see the combined effect of stripping off and using the bubble. Two simulation cases are compared: with and without bubble. 


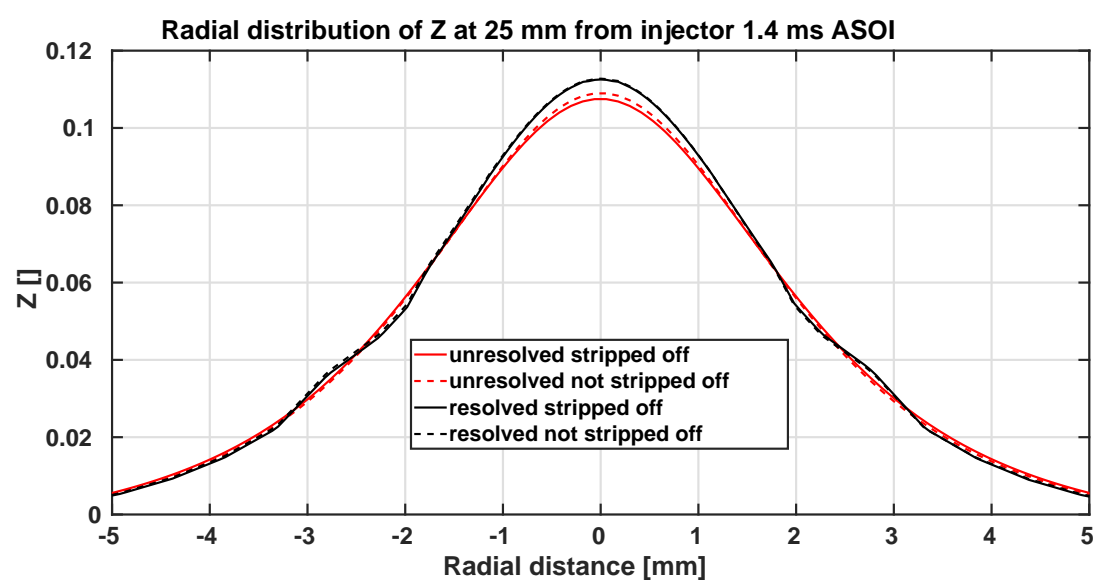

(a)

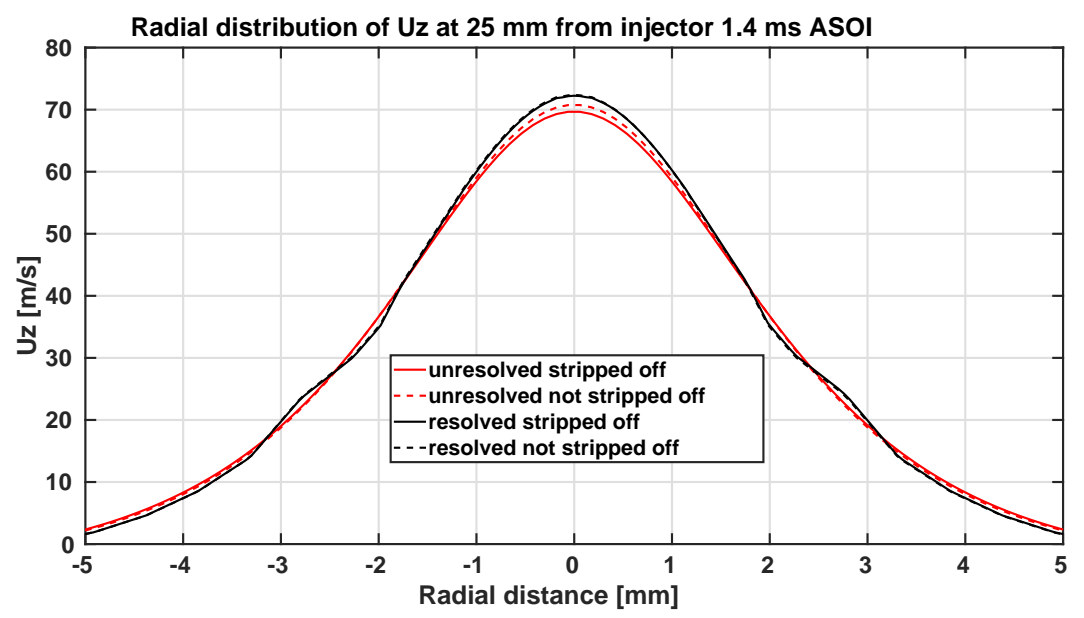

(b)

FIG. 35: Mixture fraction and velocity radial profile at $1.4 \mathrm{~ms}$ ASOI. Radial profile of (a) mixture and (b) axial fractions.

\subsection{Liquid and Vapor Penetration}

The liquid and vapor penetration for the two cases are shown in Fig. 36. Although the difference in liquid penetration is small, it is seen that the liquid penetration is relatively higher for the case with bubble. This is because the bubble volume controls the evaporation rate rather than the grid cell.

\subsection{Evaporation Rate and SMD}

The evaporation rates for the two cases are shown in Fig. 37. We see (especially from the zoomed plot) that the evaporation rate is more controlled since the start of injection for the case with bubble. The controlled evaporation rate means that the stripped off liquid droplets stay longer in the system.

Volume 31, Issue 4, 2021 


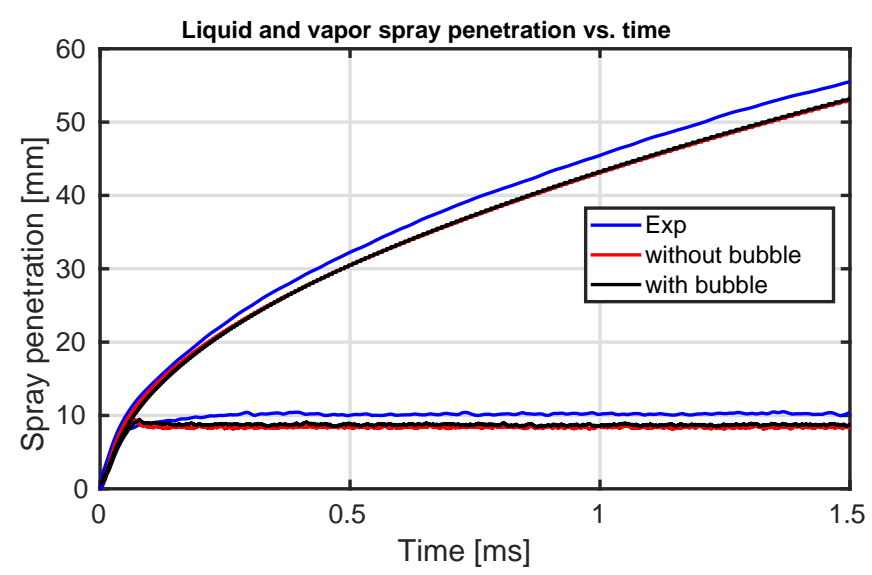

FIG. 36: Liquid and vapor penetration for the unresolved mesh

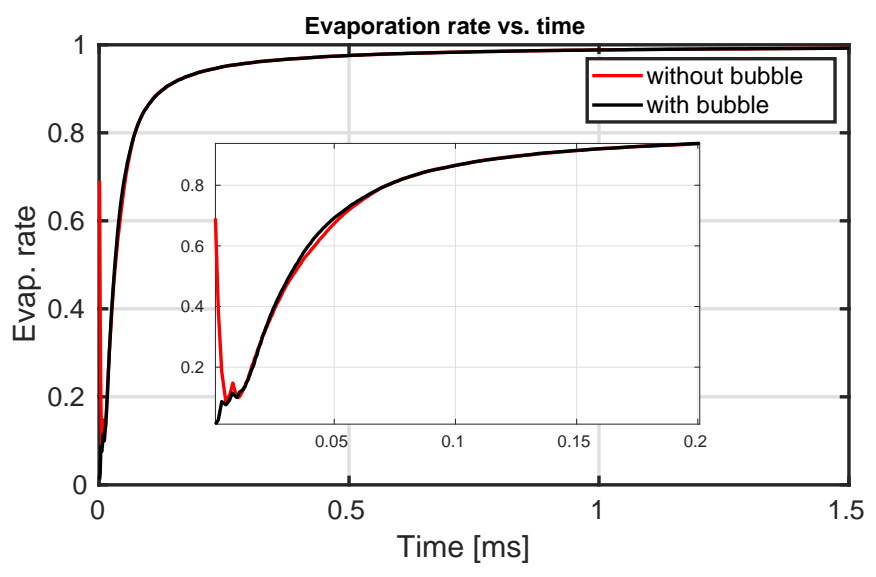

FIG. 37: Evaporation rate for unresolved orifice mesh

\subsection{Lagrangian Spray}

The Lagrangian spray for both cases are shown in Fig. 38, and the glyph of the spray sized by blob mass is shown in Fig. 39. Although the difference is small, the controlled effect of evaporation on including the bubble can be seen. The case with bubble has a relatively higher number of smaller droplets.

It can thus be concluded that although the difference is small, including the bubble produces a more controlled evaporation as the liquid droplets now interact with the bubble rather than the grid cell. It is expected that the influence of bubble would be stronger when a coarser mesh is used.

\section{NUMERICAL UNCERTAINTY}

The numerical uncertainty obtained by solution verification and quantified by GCI presented earlier for the key variables (Section 5) for one specific point in time is now plotted as an error 


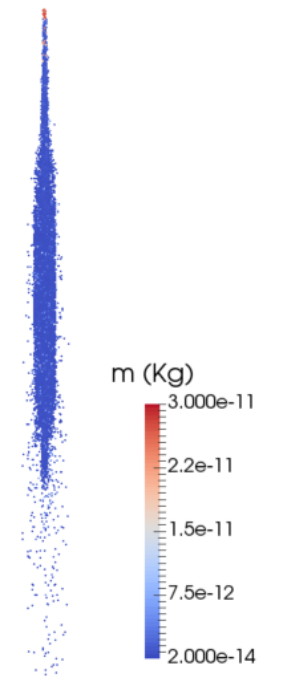

(a)

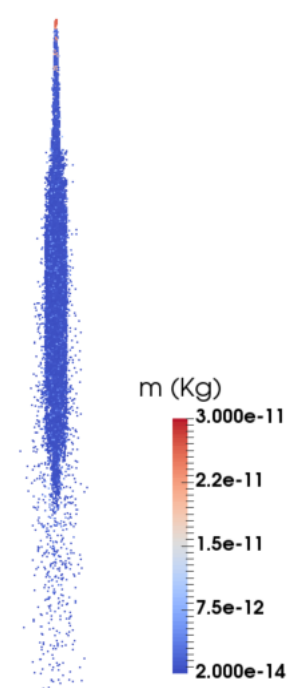

(b)

FIG. 38: Lagrangian spray for the unresolved mesh colored according to droplet mass. (a) Without bubble and (b) with bubble.

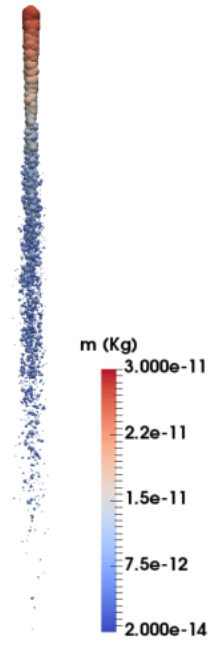

(a)

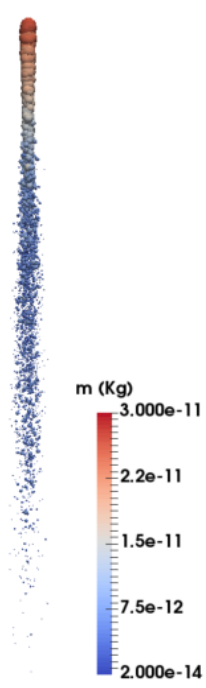

(b)

FIG. 39: Glyph representation of spray for the unresolved mesh sized according to blob mass. (a) Without bubble and (b) with bubble.

bar (Fig. 40) for the resolved mesh and compared against experimental data and the unresolved mesh (including stripping off of droplets and influence of bubble). This gives an estimate of both how much the numerical uncertainty is and how well the model validates with the experiment within the uncertainty. It is seen that even within the uncertainty the simulation data agrees reasonably with that of the experiment.

Volume 31, Issue 4, 2021 


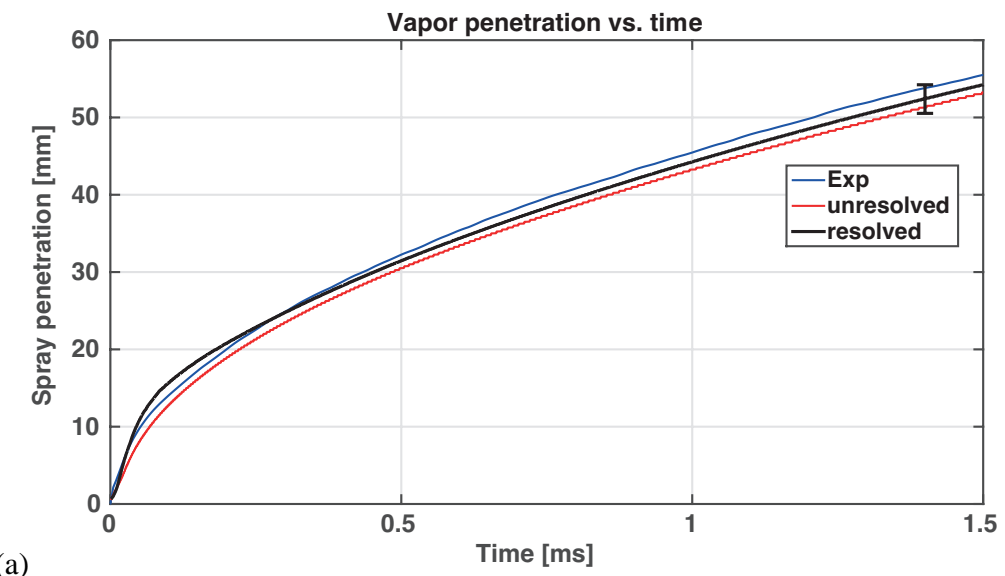

(a)

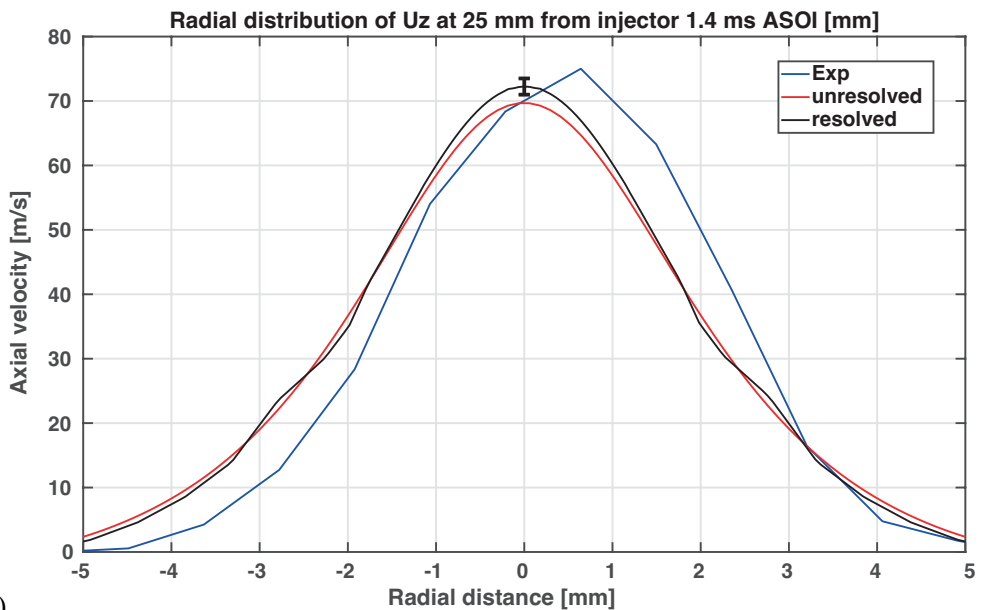

(b)

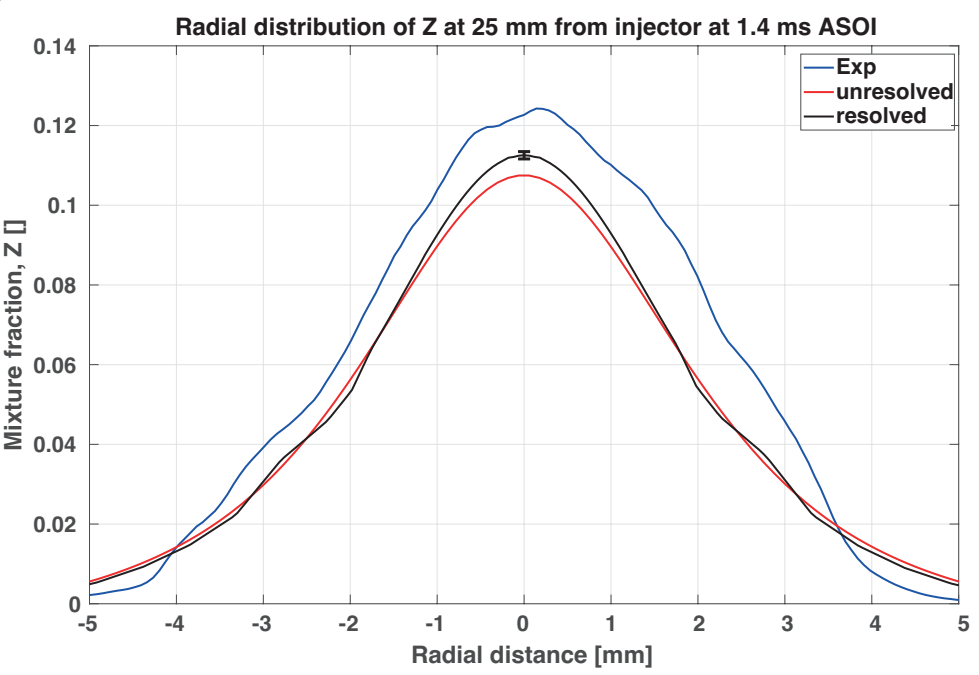

FIG. 40: Numerical uncertainty of key variables. (a) Vapor penetration; radial distribution of (b) axial velocity and (c) mixture fraction. 


\section{CONCLUSIONS}

Grid resolution is an important topic discussed among the spray modeling community. There has been much debate on what constitutes adequate mesh resolution. Apart from considering grid resolution in the near orifice region, it has been shown that it is important to resolve the injector orifice itself, to improve predictions of gas jets. However, few studies have investigated resolving the injector orifice for sprays, injected under high pressure and temperature diesel engine-like conditions. In this work the injector orifice was resolved into nine cells (three cells across the diameter) and the influence on spray formation was studied. A simple solution verification showed that the fine grid used in a resolved orifice was reasonable when compared to the extrapolated values of the results for a few key variables. Two different meshes were investigated, one with and the other without resolving the injector orifice, and the simulated results were compared with the ECN spray A experimental data. The following conclusions were drawn:

- Simulated liquid and vapor penetration showed reasonably good agreement with the experiment. The liquid and vapor penetration values of the resolved mesh were slightly higher than those of the unresolved mesh, and vapor penetration of the resolved mesh was closer to that from the experiment.

- When simulating an asymmetrical input injection velocity, fine asymmetrical structures at the leading edge of the spray were observed in the resolved orifice mesh but not in the unresolved mesh.

- Turbulence (measured by $k$ and $\epsilon$ values) and the mixing rate of fuel with air were higher for the resolved mesh than the unresolved mesh, especially for the rich region. The mixing rate is an important parameter to consider when studying combustion.

Overall, it can be concluded that resolving the injector orifice has an influence on the predicted spray formation. Moreover, it was shown that it is possible to perform spray simulations with the Eulerian-Lagrangian method for fine grid cells ( $28 \mu \mathrm{m}$ in the $x-y$ plane in the injector orifice). This work was intended to give an insight into the influence of resolving the injector orifice, but final comment can be drawn after studying the influence of the spray for combusting conditions. Moreover, it should also be noted that resolving the injector orifice requires the change of the mesh itself; a cylindrical core is needed for the resolved mesh in order to allow for a gradual transition in grid cells from the core (spray region) to the periphery. The results therefore represent the combined effect of resolving the injector orifice and the overall fine mesh used. And for the unresolved mesh it was intended to use a cartesian core for all the comparisons as this is typically seen for spray simulations. However, a short study was also made on the difference of results when using a cartesian core over a cylindrical core for the unresolved mesh. It will be interesting in the future to further investigate the unresolved mesh with cylindrical core.

The influence of creating new child blobs from the mass stripped off from a parent blob by secondary breakup was investigated for both resolved and unresolved injector orifices and the following conclusions were drawn:

- There was a small difference in liquid penetration for the unresolved orifice between the case where the stripped off droplets were retained in the parent blob and the case where new blobs were formed from stripped off droplets. The smaller droplets produced in the stripped off case heated up and evaporated faster and produced a slightly lower liquid penetration. No significant difference was observed in vapor penetration.

Volume 31, Issue 4, 2021 
- No significant differences were observed in the resolved orifice mesh for liquid and vapor penetration.

- There were also no significant differences in the radial gas velocity and mixture fraction profiles for both the resolved and unresolved meshes.

It can therefore be concluded that under high pressure and temperature diesel engine-like operating conditions, the formation of new child blobs from stripped off mass has no significant effect on spray predictions. Including stripped off blobs also requires higher CPU time. However it is worthwhile to investigate creating stripped off blobs for lower ambient evaporating conditions (ambient temperature lower than in ECN spray A).

The influence of introducing bubble was also studied and it was seen that introducing the bubble resulted in a more controlled evaporation for the unresolved mesh.

\section{ACKNOWLEDGMENTS}

The authors would like to thank the Combustion Engine Research Centre (CERC) for financing this project. The simulations were performed on resources at Chalmers Centre for Computational Science and Engineering (C3SE) provided by the Swedish National Infrastructure for Computing (SNIC).

\section{REFERENCES}

Abani, N., Kokjohn, S., Park, S., Bergin, M., Munnannur, A., Ning, W., Sun, Y., and Reitz, R.D., An Improved Spray Model for Reducing Numerical Parameter Dependencies in Diesel Engine CFD Simulations, SAE Technical Paper No. 2008-01-0970, 2008.

Abraham, J., What Is Adequate Resolution in the Numerical Computations of Transient Jets, SAE Technical Paper No. 970051, 1997.

ASME V\&V 20-2009, Standard for Verification and Validation in Computational Fluid Dynamics and Heat Transfer, New York: The American Society of Mechanical Engineers, 2009.

Computing at Lawrence Livermore National Laboratory, SUNDIALS (Suite of Nonlinear and DIfferential/ALgebraic Equation Solvers), accessed March 30, from https://computing.llnl.gov/projects/sundials/ kinsol, 2020.

Ducowicz, J.K., A Particle-Fluid Numerical Model for Liquid Sprays, J. Comput. Phys., vol. 35, pp. 229$253,1980$.

ECN, Diesel Data Search, accessed: March 30, from https://ecn.sandia.gov/ecn-data-search/, 2020.

El Wakil, M., Ueyhara, O., and Myers, F., A Theoretical Investigation of Heating-Up Period of Injected Fuel Droplets Vaporizing in Air, NACA, Tech. Rep. TN 3179, 1954.

Engine Combustion Network, ECN2 Workshop, Mixing and Velocity, Modelling, acessed June 30, from https://ecn.sandia.gov/ecn-workshop/ecn2-proceedings/, 2020.

Gosman, A.D. and Ioannides, E., Aspects of Computer Simulation of Liquid-Fueled Combustors, J. Energy, vol. 7, no. 6, pp. 482-490, 1983.

IFPEN, IFP Energies Nouvelles, accessed March 30, from https://www.ifpenergiesnouvelles.com/, 2020.

Jones, W.P. and Launder, B.E., The Prediction of Laminarization with a Two-Equation Model of Turbulence, Int. J. Heat Mass Transfer, vol. 15, no. 2, pp. 301-314, 1972.

Karlsson, A., Modelling Auto-Ignition, Flame Propagation and Combustion in Non-Stationary Turbulent Sprays, PhD, Chalmers University of Technology, 1995. 
Karlsson, A., Husberg, T., and Denbratt, I., Analysis of Advanced Multiple Injection Strategies in a HeavyDuty Diesel Engine Using Optical Measurements and CFD-Simulations, SAE Technical Paper No. 2008-01-1328, 2008.

Kösters, A. and Karlsson, A., Validation of the VSB2 Spray Model Against Spray A and Spray H, Atomization Sprays, vol. 26, no. 8, pp. 775-798, 2016.

Lippert, A.M. and Reitz, R.D., Modeling of Multicomponent Fuels Using Continuous Distributions with Application to Droplet Evaporation and Sprays, SAE Technical Paper No. 972882, 1997.

Lucchini, T., D’Errico, G., and Ettorre, D., Numerical Investigation of the Spray Mesh Turbulence Interactions for High-Pressure, Evaporating Sprays at Engine Conditions, Int. J. Heat Fluid Flow, vol. 32, no. 1, pp. 285-297, 2011.

OpenFOAM, The OpenFOAM Foundation, accessed June 30, from https://openfoam.org/, 2020.

Perini, F. and Reitz, R.D., Improved Atomization, Collision and Sub-Grid Scale Momentum Coupling Models for Transient Vaporizing Engine Sprays, Int. J. Multiphase Flow, vol. 79, pp. 107-123, 2016.

Pilch, M. and Erdman, C.A., Use of Breakup Time Data and Velocity History Data to Predict the Maximum Size of Stable Fragments for Acceleration-Induced Breakup of a Liquid Drop, Int. J. Multiphase Flow, vol. 13, no. 6, pp. 741-757, 1987.

Ra, Y. and Reitz, R.D., A Vaporization Model for Discrete Multi-Component Fuel Sprays, Int. J. Multiphase Flow, vol. 35, no. 2, pp. 101-117, 2009.

Roache, P.J., Verification and Validation in Computational Science and Engineering, Albuquerque: Hermosa Publishers, 1998.

Richardson, L.F., The Approximate Arithmetical Solution by Finite Differences of Physical Problems Involving Differential Equations, with an Application to the Stresses in a Mansory Dam, Transact. R. Soc. London, Ser. A, vol. 210, pp. 307-357, 1910.

Schmehl, R., Maier, G., and Wittig, S., CFD Analysis of Fuel Atomization, Secondary Droplet Breakup and Spray Dispersion in the Premix Duct of a LPP Combustor, Eight Int. Conf. on Liquid Atomization and Spray Systems, Pasadena, CA, 2000.

Senecal, P.K., Pomraning, E., Richards, K.J., and Som, S., Grid-Convergent Spray Models for Internal Combustion Engine CFD Simulations, Proc. of the ASME 2012 Internal Combustion Engine Division Fall Technical Conference, pp. 697-710, 2012.

Weller, H.G., Tabor, G., Jasak, H., and Fureby, C., A Tensorial Approach to Computational Continuum Mechanics Using Object-Oriented Techniques, Comput. Phys., vol. 12, no. 6, pp. 620-631, 1998.

Volume 31, Issue 4, 2021 